\title{
Antitrypanosomal Lactones from Nectandra barbellata
}

Vinicius S. Londero, ${ }^{\dagger}$ Thais A. Costa-Silva, ${ }^{\ddagger}$ Guilherme M. Antar ${ }^{\perp}$ João B. Baitello, II Larissa V. F. de Oliveira, ${ }^{\dagger}$ Fernanda F. Camilo, ${ }^{\dagger}$ Andrea N. L. Batista, ${ }^{\#}$ João M. Batista Jr., ${ }^{\pi}$ Andre G. Tempone,,${ }^{,}$ João Henrique G. Lago¥,*

†Institute of Environmental, Chemical and Pharmaceutical Sciences, Federal University of São Paulo, São Paulo, Brazil.

$\ddagger$ Center for Natural and Human Sciences, Federal University of ABC, São Paulo, Brazil.

${ }^{\perp}$ Department of Botany, Institute of Biosciences, University of São Paulo, São Paulo, Brazil.

\|Dasonomy Division, Instituto Florestal, São Paulo, Brazil.

\# Institute of Chemistry, Fluminense Federal University, Rio de Janeiro, Brazil. "Institute of Science and Technology, Federal University of São Paulo, São Paulo, Brazil.

§ Centre for Parasitology and Mycology, Instituto Adolfo Lutz, São Paulo, Brazil.

${ }^{*}$ Corresponding authors.

Tel: +55 11 4996-7960; E-mail: joao.lago@ufabc.edu.br (J.H.G.L.)

Tel: +55 11 3068-2803; E-mail: andre.tempone@ial.sp.gov.br (A.G.T.) 


\section{SUPPORTING INFORMATION}

Figure S1. Positive mode ESI-qTOF-MS of compound 1.

Figure S2. ${ }^{1} \mathrm{H}$ NMR spectrum $\left(500 \mathrm{MHz}, \mathrm{CDCl}_{3}\right)$ of compound 1 .

Figure S3. ${ }^{13} \mathrm{C}$ NMR spectrum (125 MHz, $\mathrm{CDCl}_{3}$ ) of compound 1 .

Figure S4. COSY spectrum (500 MHz, $\left.\mathrm{CDCl}_{3}\right)$ of compound 1.

Figure S5. HSQC spectrum (500 and $125 \mathrm{MHz}, \mathrm{CDCl}_{3}$ ) of compound 1.

Figure S6. $\mathrm{HMBC}$ spectrum (500 and $125 \mathrm{MHz}, \mathrm{CDCl}_{3}$ ) of compound 1.

Figure S7. NOESY spectrum (500 MHz, $\left.\mathrm{CDCl}_{3}\right)$ of compound 1.

Figure S8. Infrared (IR) spectrum of compound 1.

Figure S9. Positive mode ESI-qTOF-MS of compound 2.

Figure S10. ${ }^{1} \mathrm{H}$ NMR spectrum (500 MHz, $\mathrm{CDCl}_{3}$ ) of compound 2.

Figure S11. ${ }^{13} \mathrm{C}$ NMR spectrum (125 MHz, $\mathrm{CDCl}_{3}$ ) of compound 2.

Figure S12. COSY spectrum ( $500 \mathrm{MHz}, \mathrm{CDCl}_{3}$ ) of compound 2.

Figure S13. $\mathrm{HSQC}$ spectrum (500 and $125 \mathrm{MHz}, \mathrm{CDCl}_{3}$ ) of compound 2.

Figure S14. $\mathrm{HMBC}$ spectrum (500 and $125 \mathrm{MHz}, \mathrm{CDCl}_{3}$ ) of compound 2.

Figure S15. NOESY spectrum ( $500 \mathrm{MHz}, \mathrm{CDCl}_{3}$ ) of compound 2.

Figure S16. Infrared (IR) spectrum of compound 2.

Figure S17. Positive mode ESI-qTOF-MS of compound 3.

Figure S18. ${ }^{1} \mathrm{H}$ NMR spectrum $\left(500 \mathrm{MHz}, \mathrm{CDCl}_{3}\right)$ of compound 3.

Figure S19. ${ }^{13} \mathrm{C}$ NMR spectrum (125 MHz, $\mathrm{CDCl}_{3}$ ) of compound 3 .

Figure S20. COSY spectrum $\left(500 \mathrm{MHz}, \mathrm{CDCl}_{3}\right)$ of compound 3.

Figure S21. $\mathrm{HSQC}$ spectrum (500 and $125 \mathrm{MHz}, \mathrm{CDCl}_{3}$ ) of compound 3.

Figure S22. $\mathrm{HMBC}$ spectrum (500 and $125 \mathrm{MHz}, \mathrm{CDCl}_{3}$ ) of compound 3.

Figure S23. NOESY spectrum ( $\left.500 \mathrm{MHz}, \mathrm{CDCl}_{3}\right)$ of compound 3. 
Figure S24. Infrared (IR) spectrum of compound 3.

Figure S25. Comparison of the observed UV and ECD spectra of (-)-2 (red trace), recorded in MeOG, with the calculated [CAMB3LYP/PCM(MeOH)/TZVP//B3PW91/PCM(MeOH)/6-311G(d,p)] UV and ECD spectra (black trace) of the simple average of the lowest-energy conformers identified for the $(R)-\mathbf{2}$.

Figure S26. Comparison of the observed IR and VCD spectra of (-)-2 (black trace), recorded in $\mathrm{CDCl}_{3}$, with the calculated [B3LYP/PCM(CHCl 3$) / 6-31 \mathrm{G}(\mathrm{d})$ ] IR and VCD spectra of the simple average of the lowest-energy conformers identified for the $(R)$-truncated model (blue trace) and $(R)-2$ (red trace).

Figure S27. Optimized structures of the lowest-energy conformers identified for $(R)-2$ at the B3LYP/PCM $\left(\mathrm{CHCl}_{3}\right) / 6-31 \mathrm{G}(\mathrm{d})$ level and used as a simple average. Figure S28. Drug-likeness of compounds $\mathbf{1}-\mathbf{3}$ using the Bioavailability Radar (SwissADME).

Table S1. Thermochemical analysis of the $(R)$-truncated model.

Table S2. Thermochemical analysis of $(R)-\mathbf{2}$.

Table S3. In silico predictions of ADME and drug-likeness of compounds 1 - $\mathbf{3}$ using SwissADME platform. 


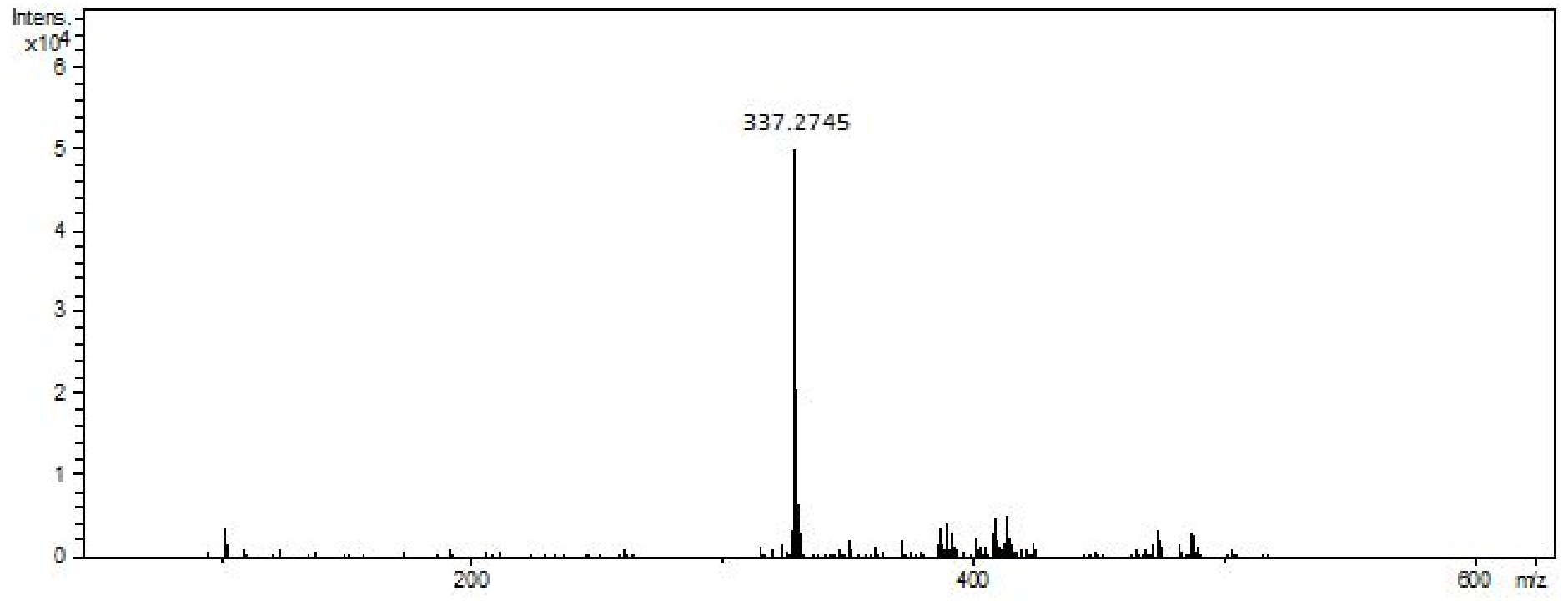

Figure S1. Positive mode ESI-qTOF-MS of compound 1. 


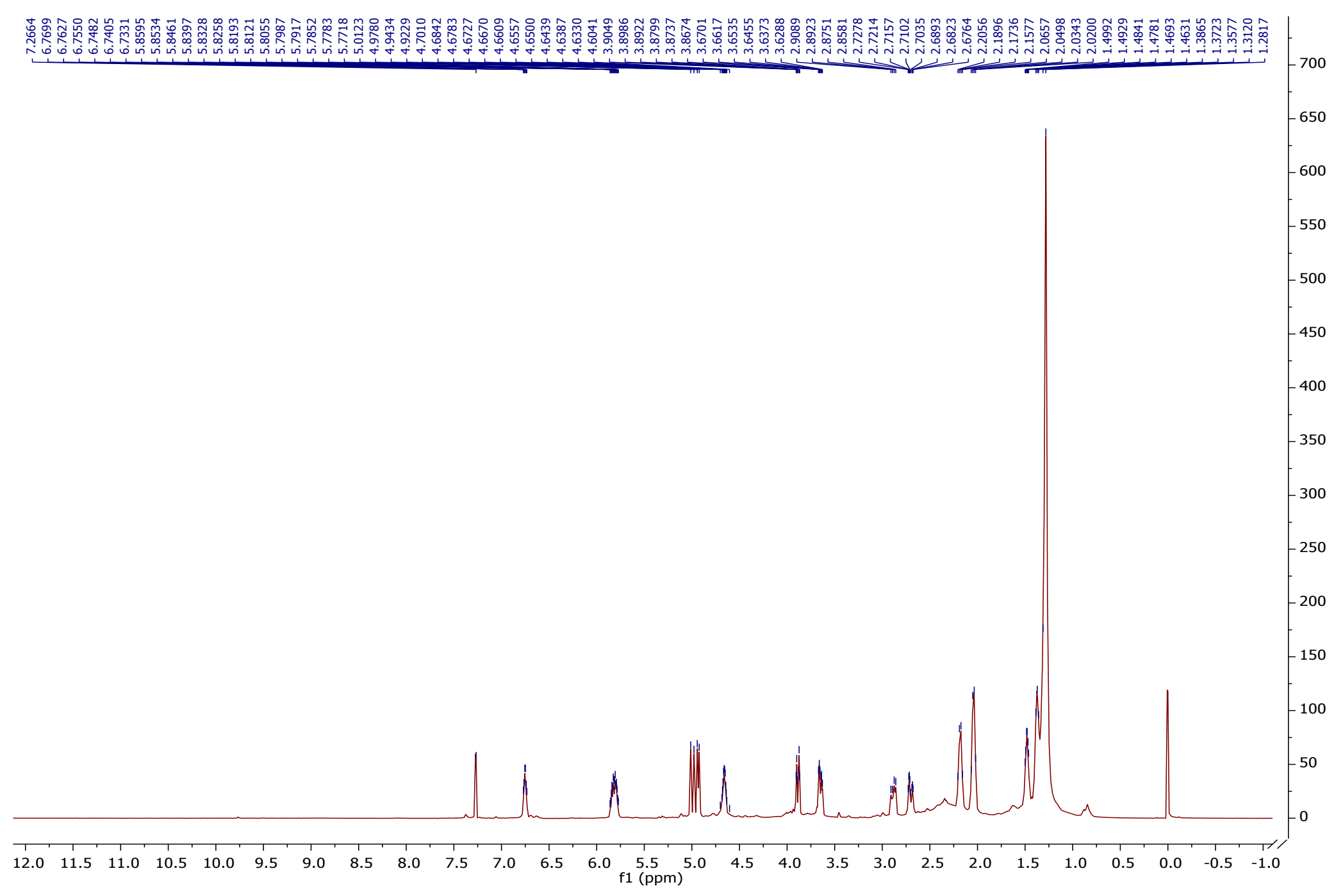

Figure S2. ${ }^{1} \mathrm{H}$ NMR spectrum $\left(500 \mathrm{MHz}, \mathrm{CDCl}_{3}\right)$ of compound 1. 


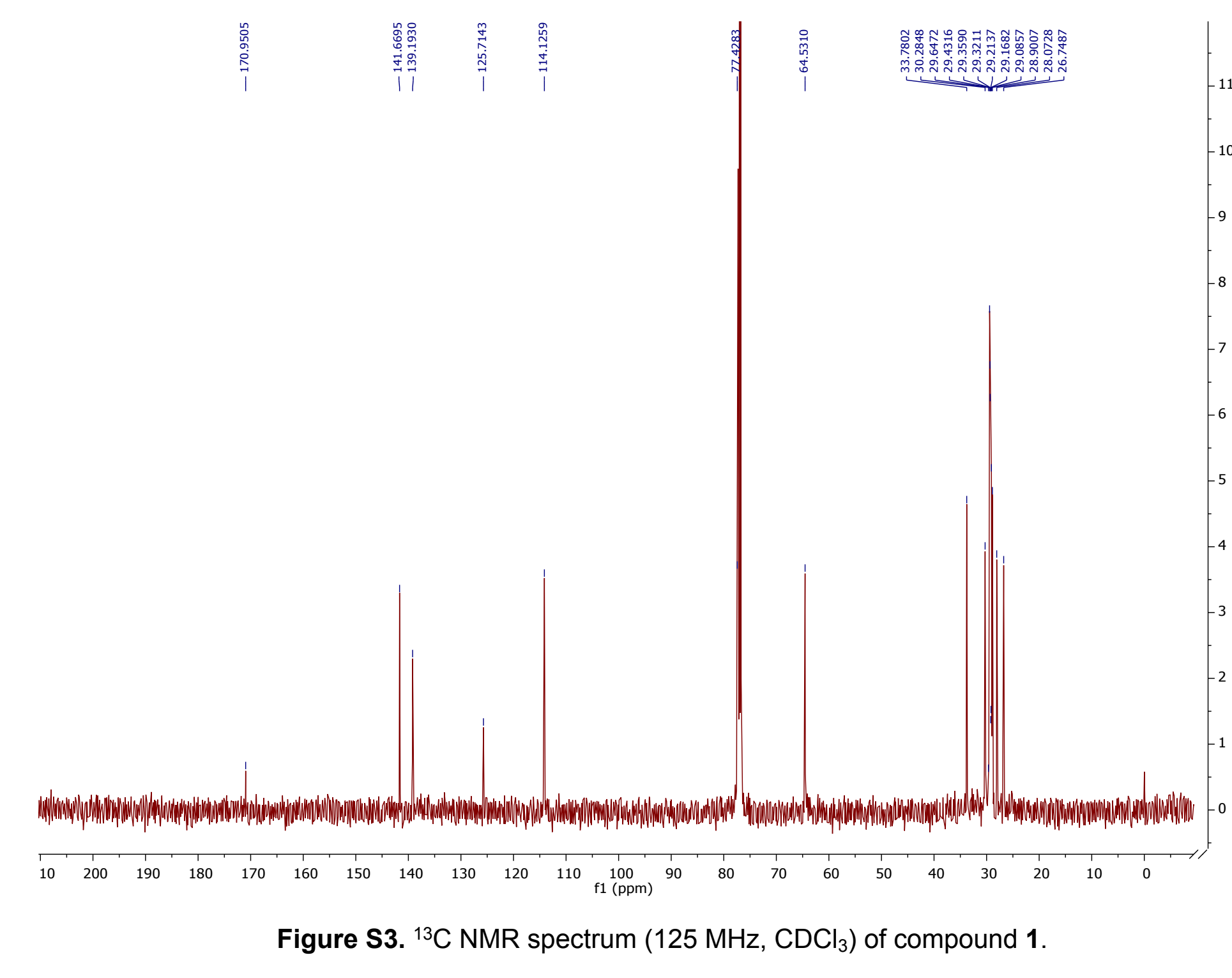




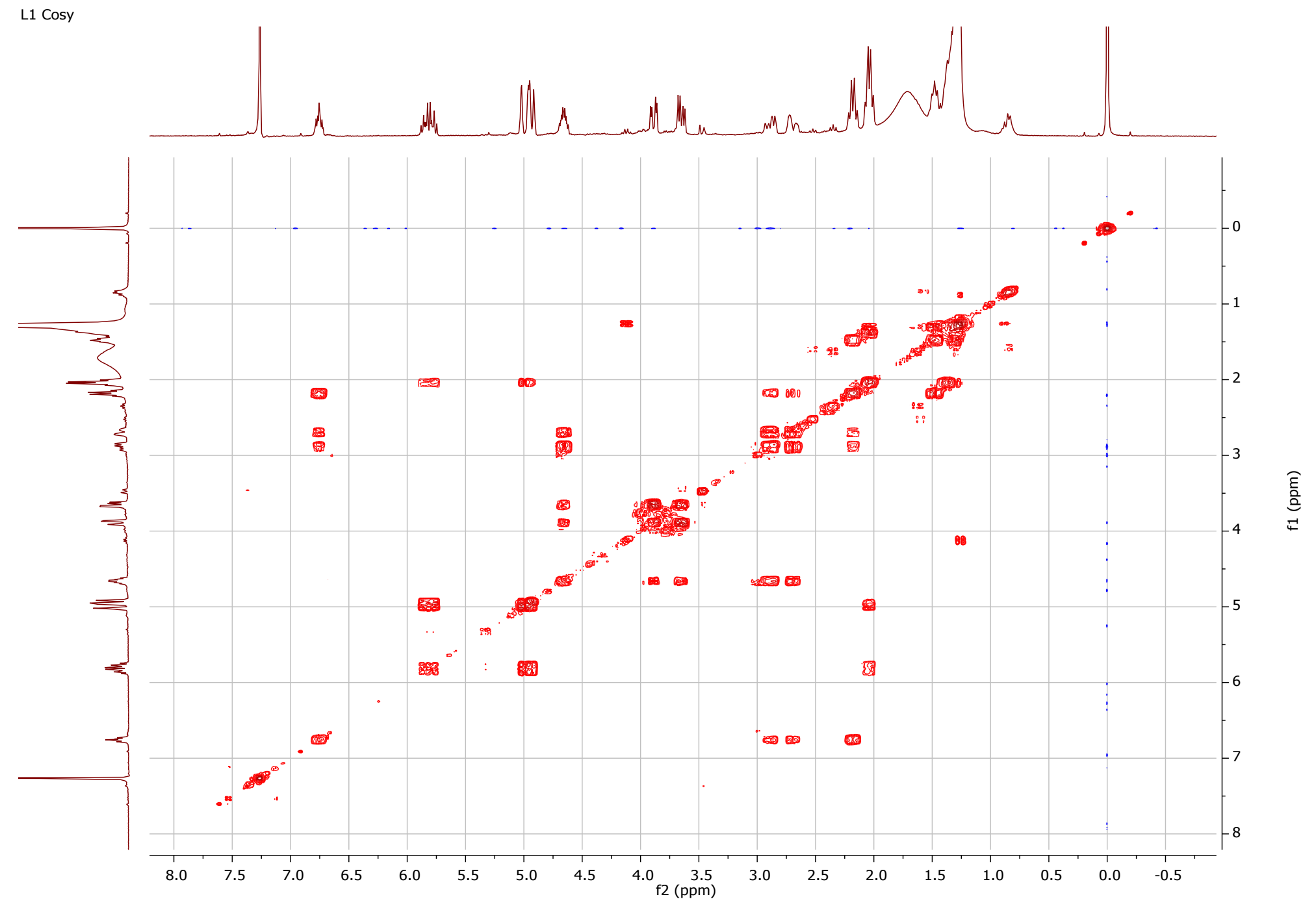

Figura S4. COSY spectrum (500 MHz, $\left.\mathrm{CDCl}_{3}\right)$ of compound 1. 


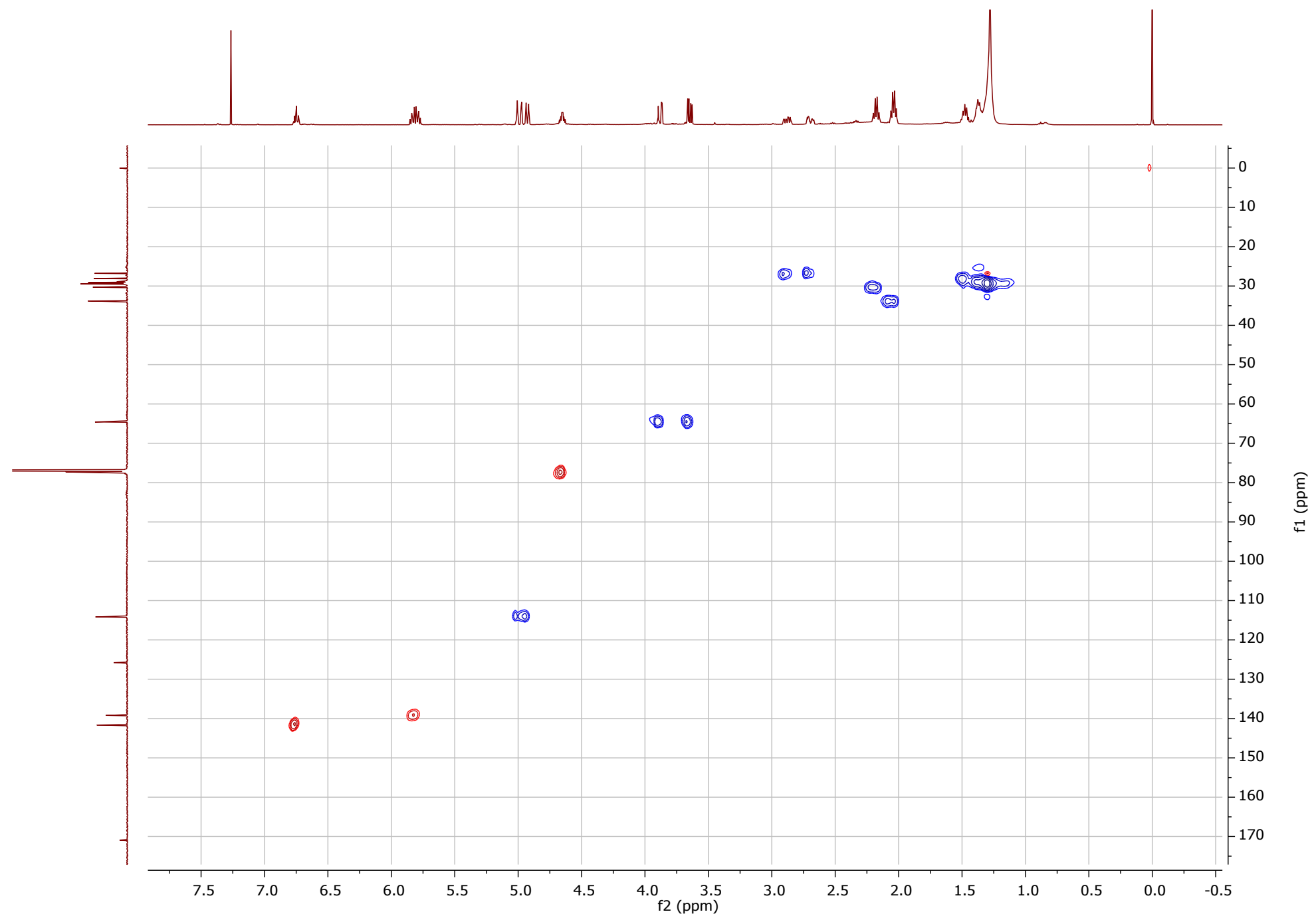

Figure S5. HSQC spectrum (500 and $125 \mathrm{MHz}_{\mathrm{CDCl}}$ ) of compound 1. 


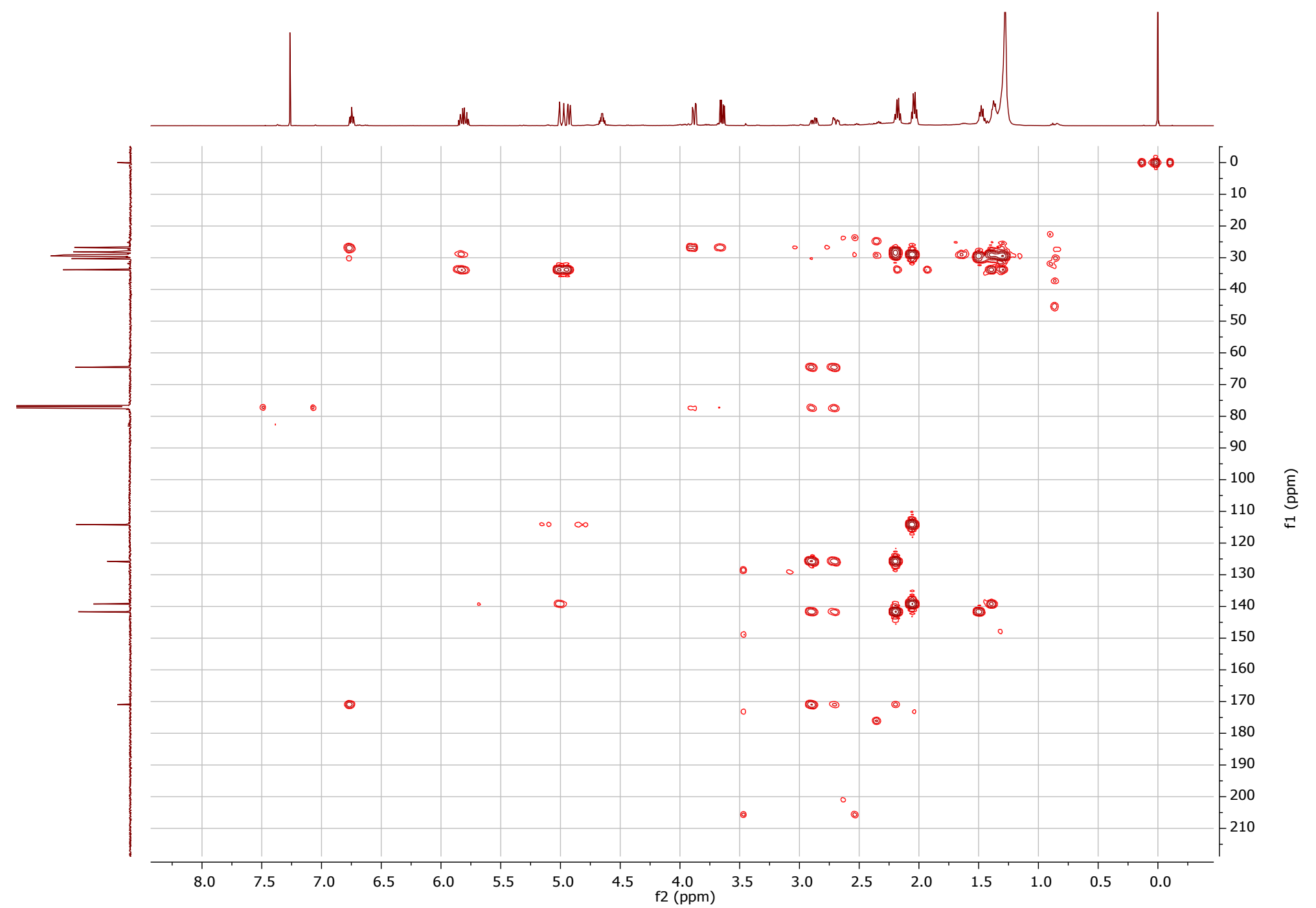

Figure S6. $\mathrm{HMBC}$ spectrum (500 and $125 \mathrm{MHz}, \mathrm{CDCl}_{3}$ ) of compound 1. 
S10

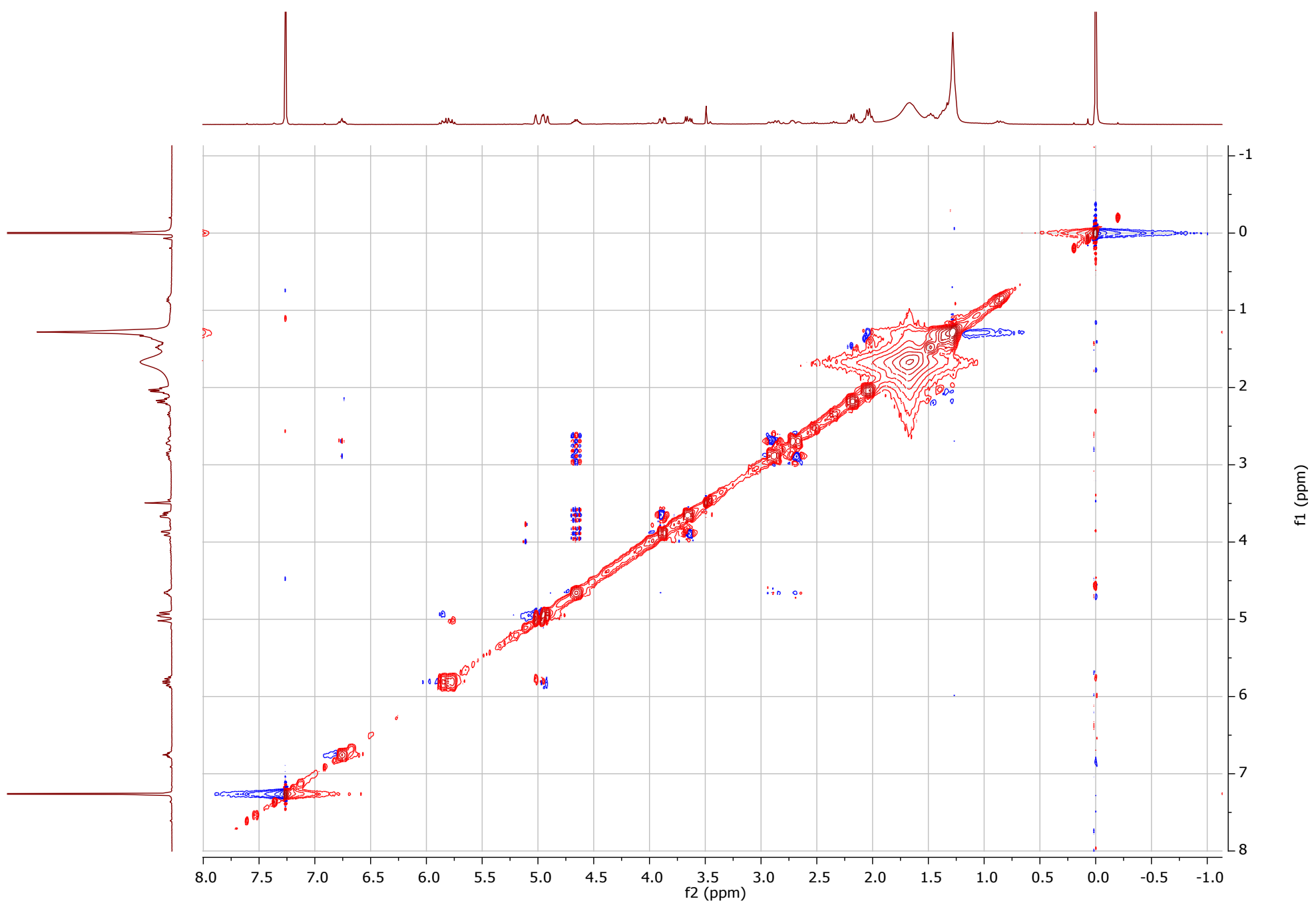

Figure S7. NOESY spectrum (500 MHz, $\mathrm{CDCl}_{3}$ ) of compound 1. 


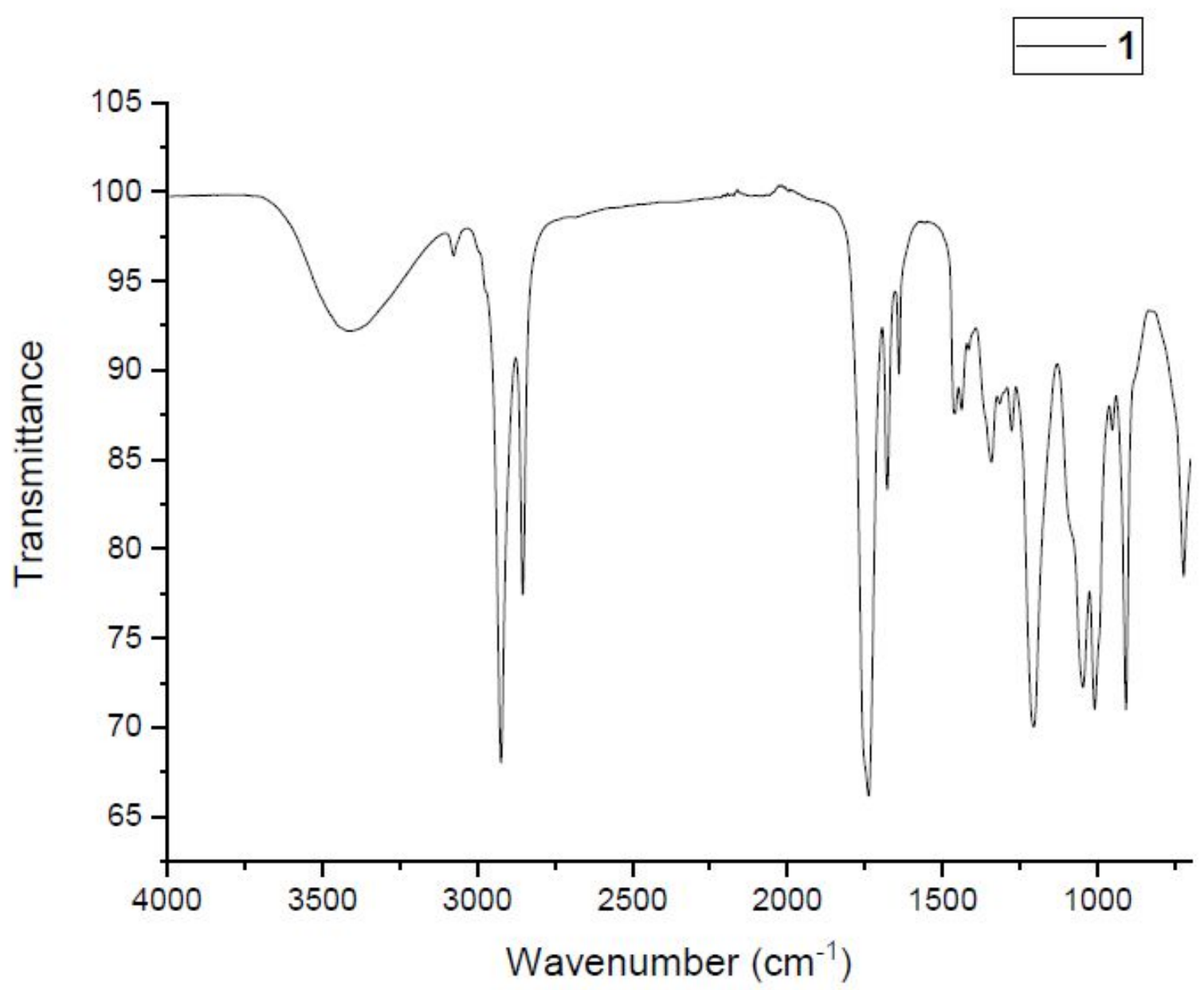

Figure S8. Infrared (IR) spectrum of compound 1. 


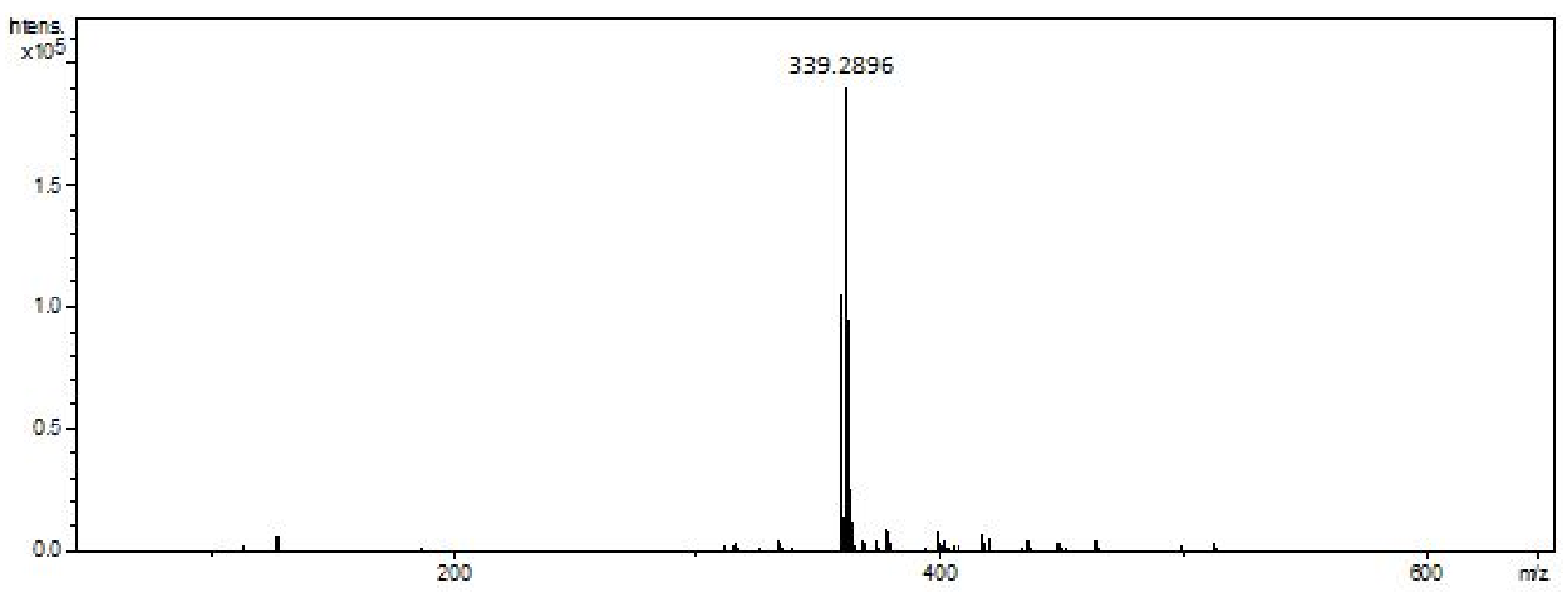

Figure S9. Positive mode ESI-qTOF-MS of compound 2. 


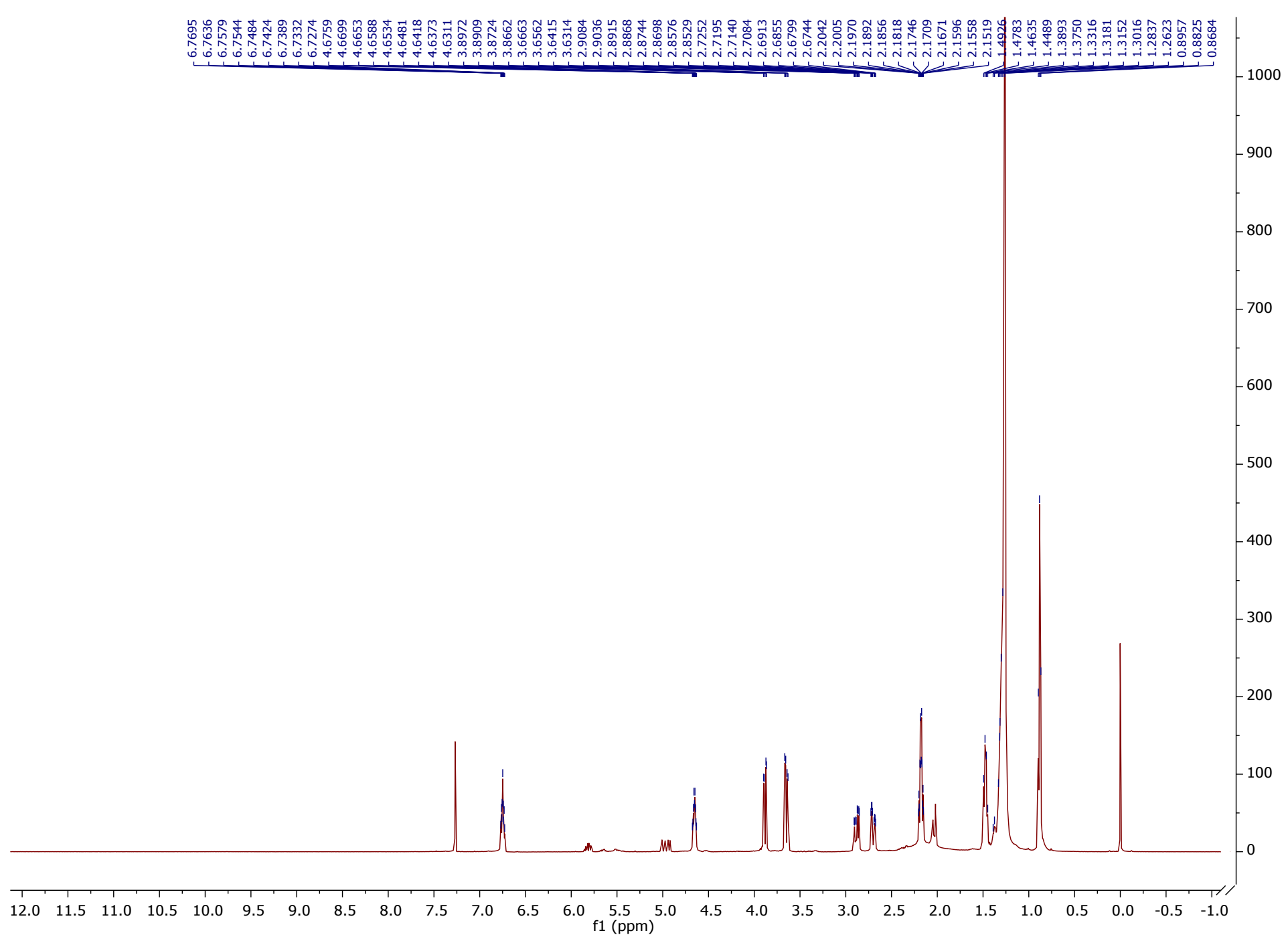

Figure S10. ${ }^{1} \mathrm{H}$ NMR spectrum $\left(500 \mathrm{MHz}, \mathrm{CDCl}_{3}\right)$ of compound 2 . 


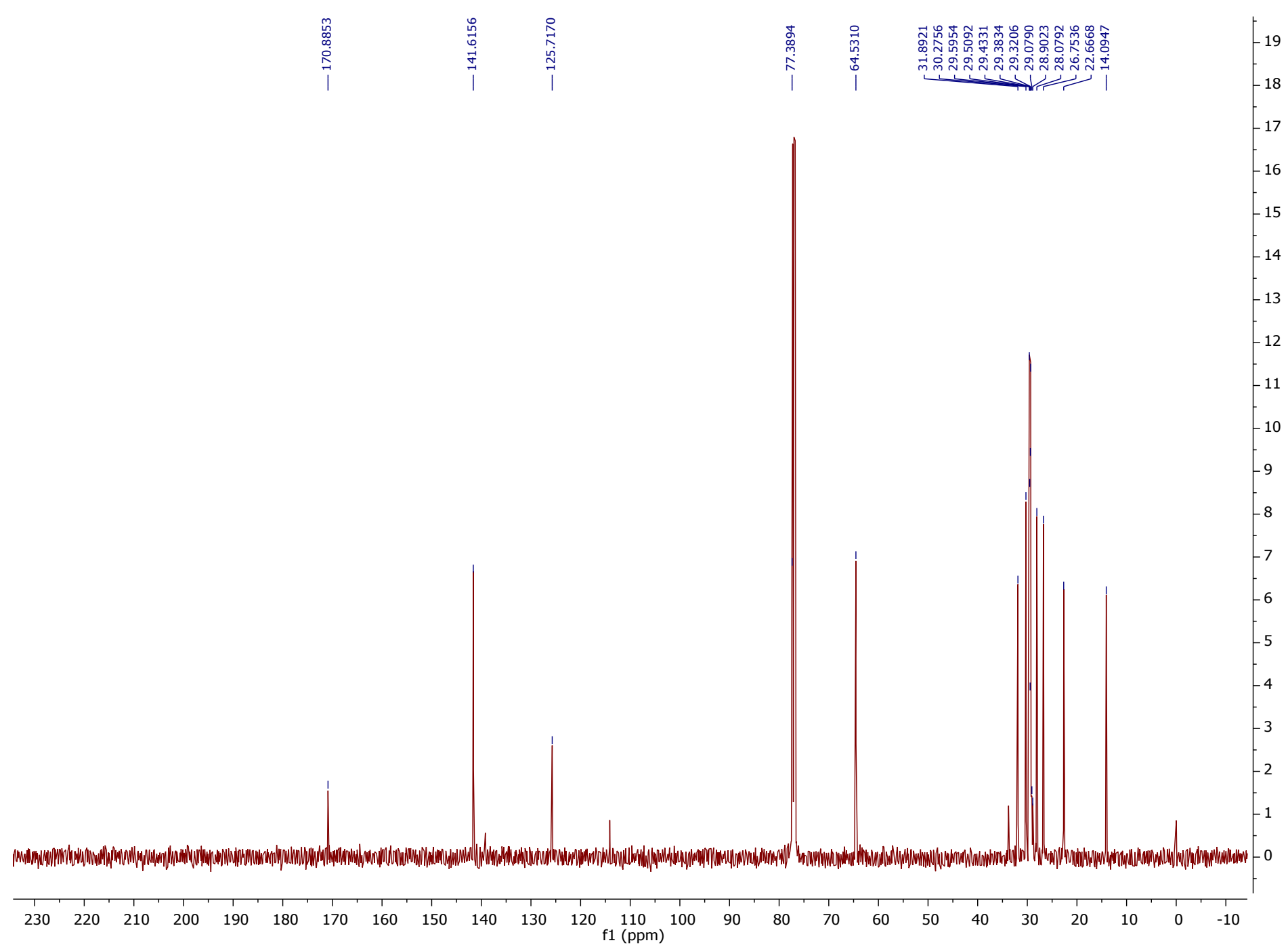

Figure S11. ${ }^{13} \mathrm{C}$ NMR spectrum $\left(125 \mathrm{MHz}, \mathrm{CDCl}_{3}\right.$ ) of compound 2. 


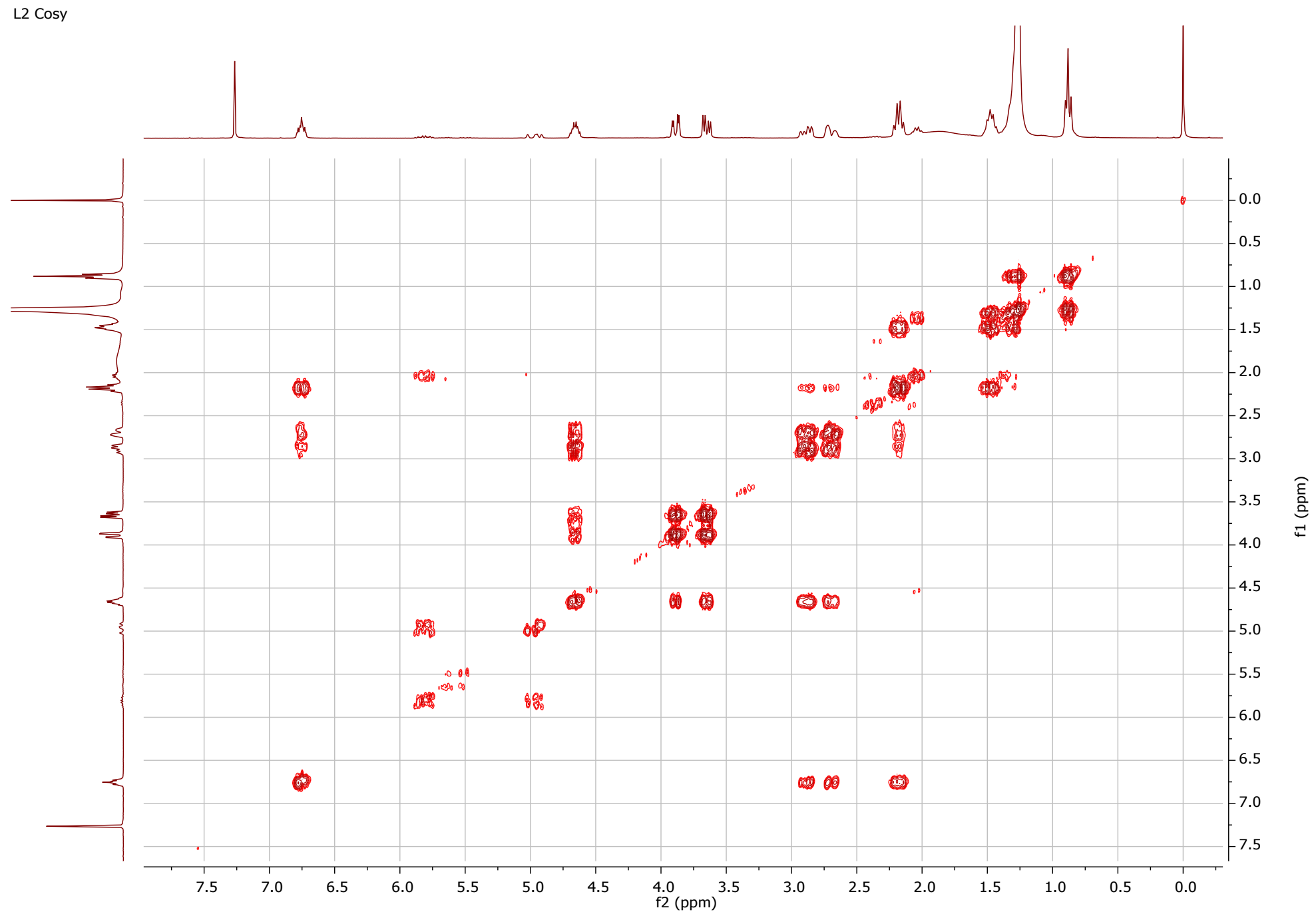

Figure S12. COSY spectrum (500 MHz, CDCl ${ }_{3}$ ) of compound 2. 


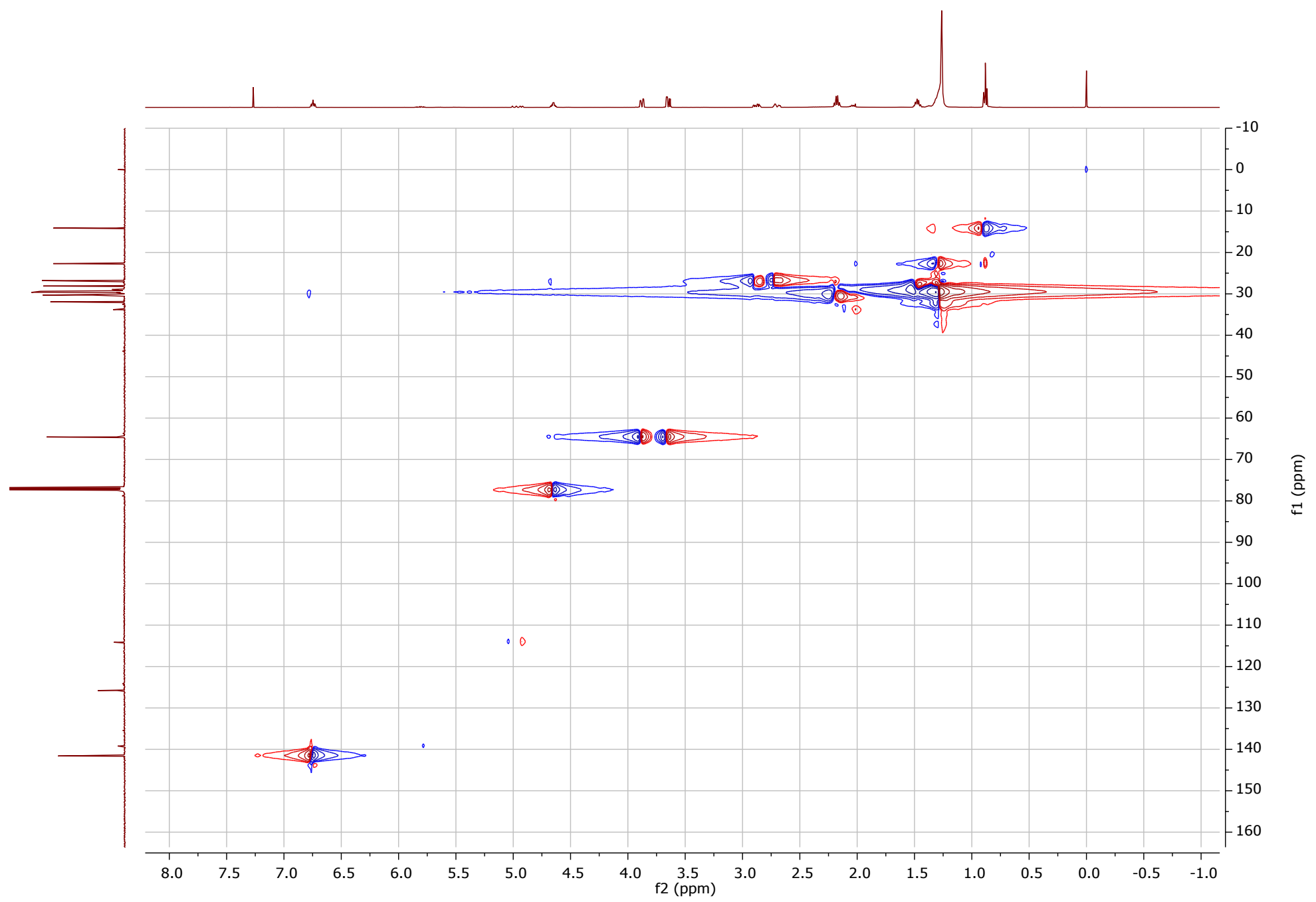

Figure S13. HSQC spectrum (500 and $125 \mathrm{MHz}, \mathrm{CDCl}_{3}$ ) of compound 2. 


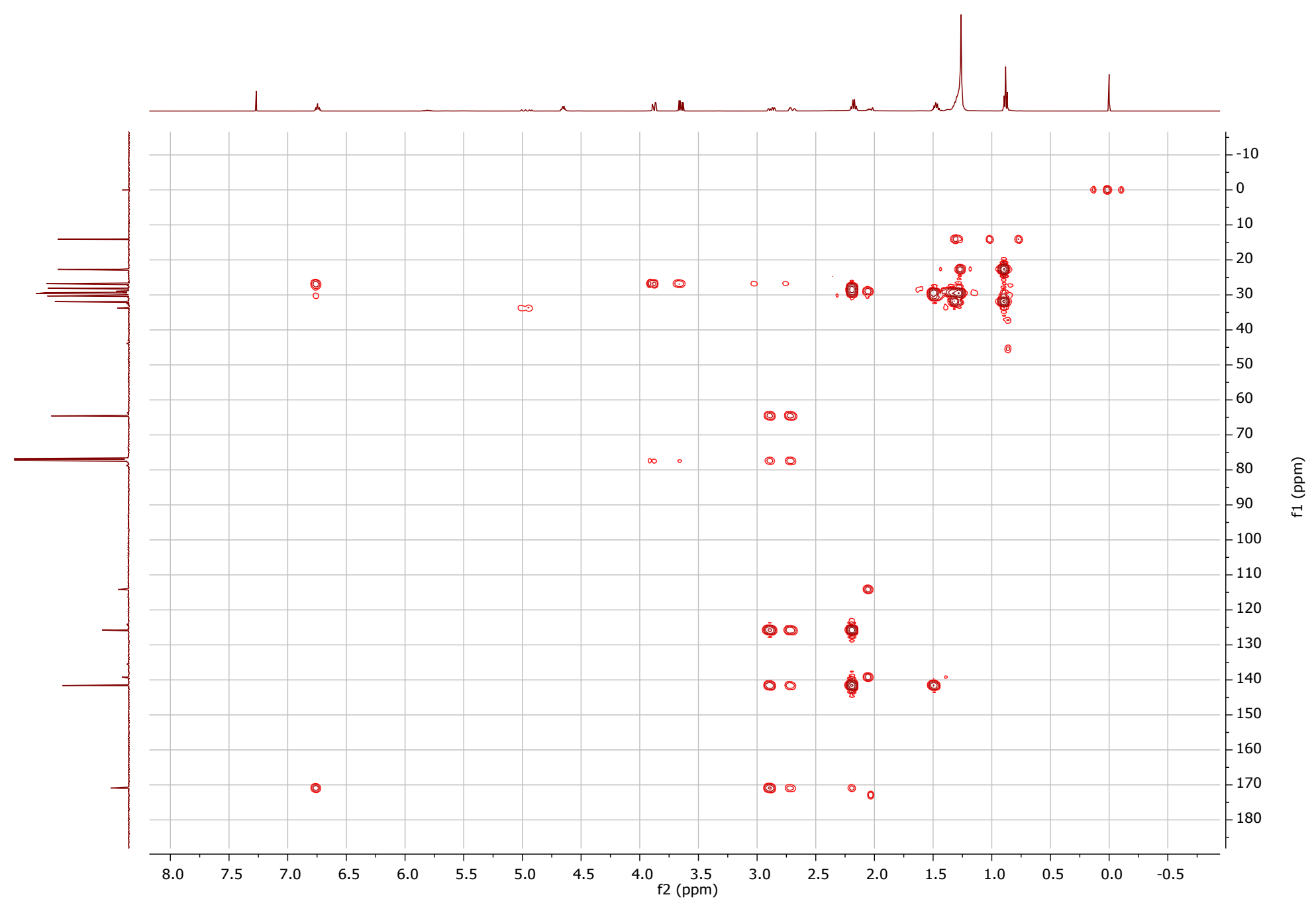

Figure S14. HMBC spectrum (500 and $125 \mathrm{MHz}, \mathrm{CDCl}_{3}$ ) of compound 2. 
<smiles>CC(C)C</smiles> 


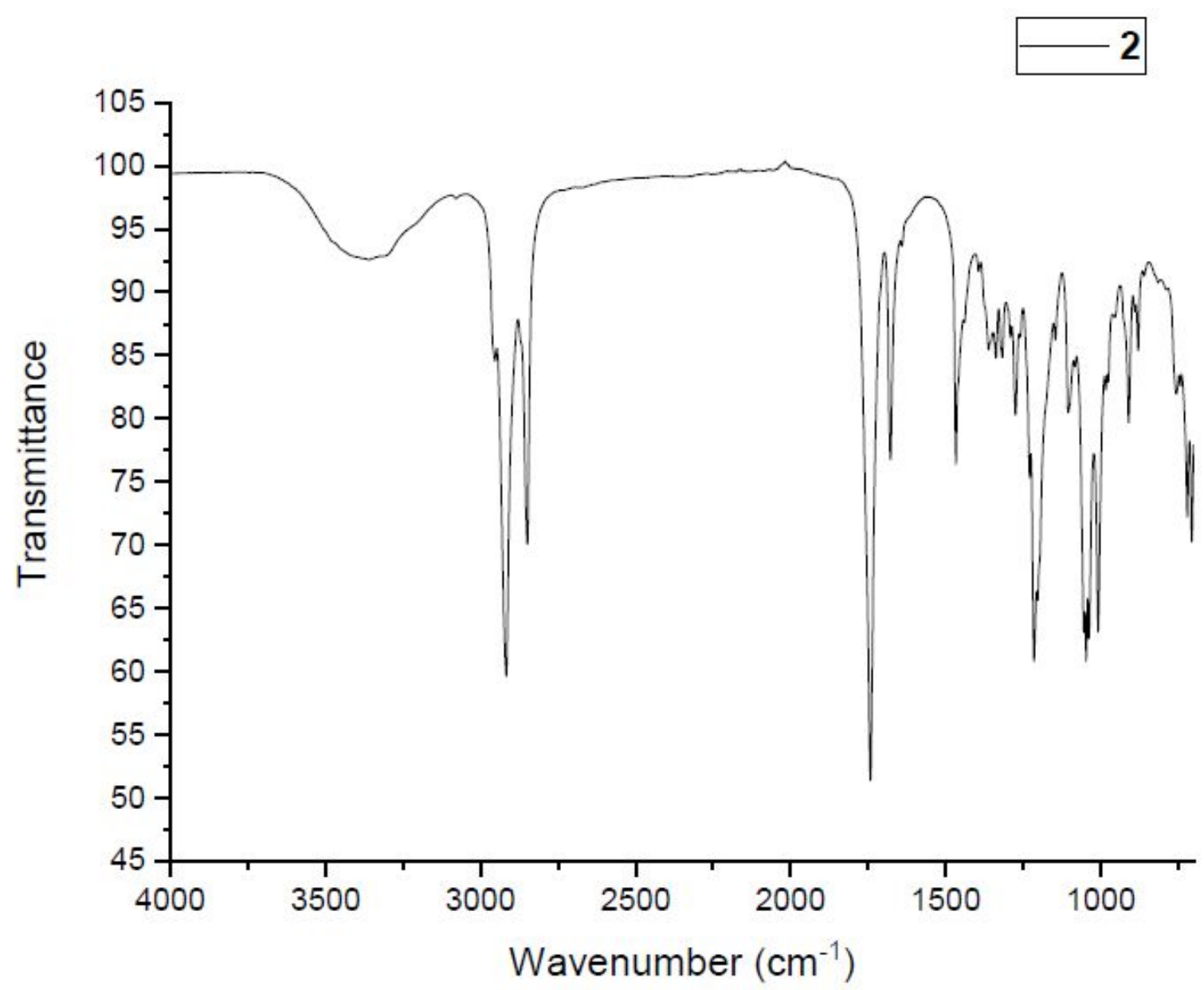

Figure S16. Infrared (IR) spectrum of compound 2. 


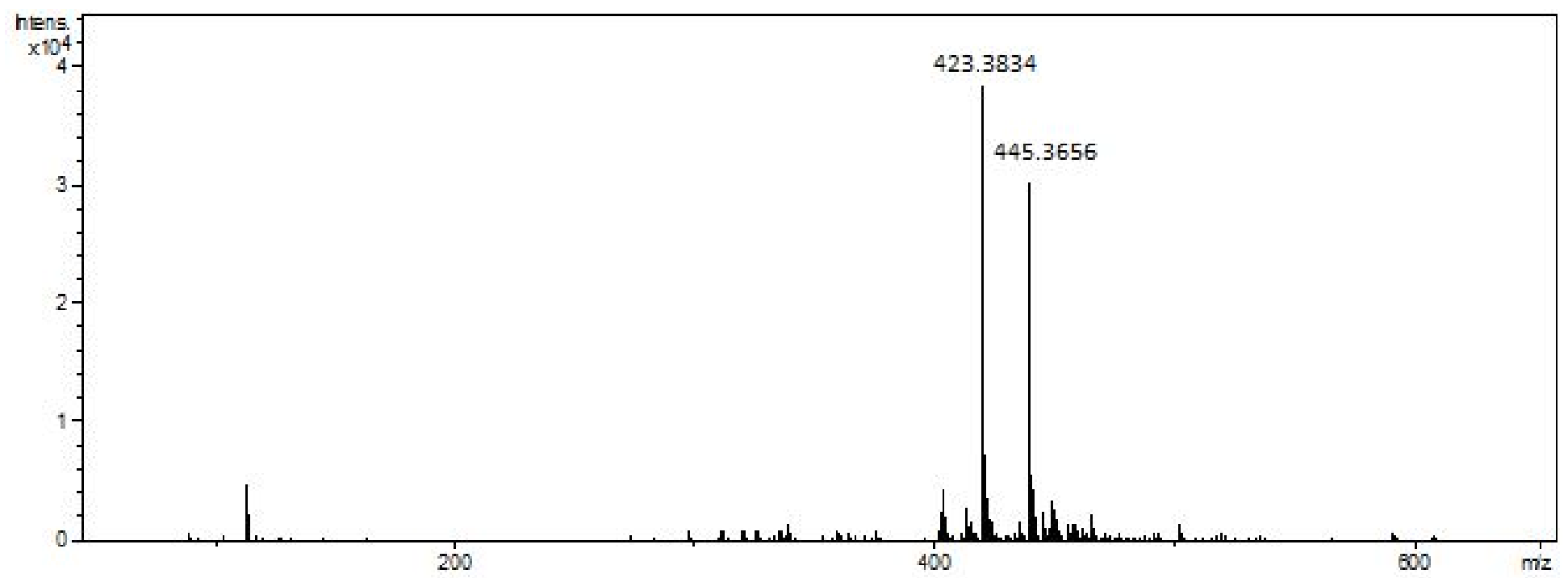

Figure S17. Positive mode ESI-qTOF-MS of compound 3 


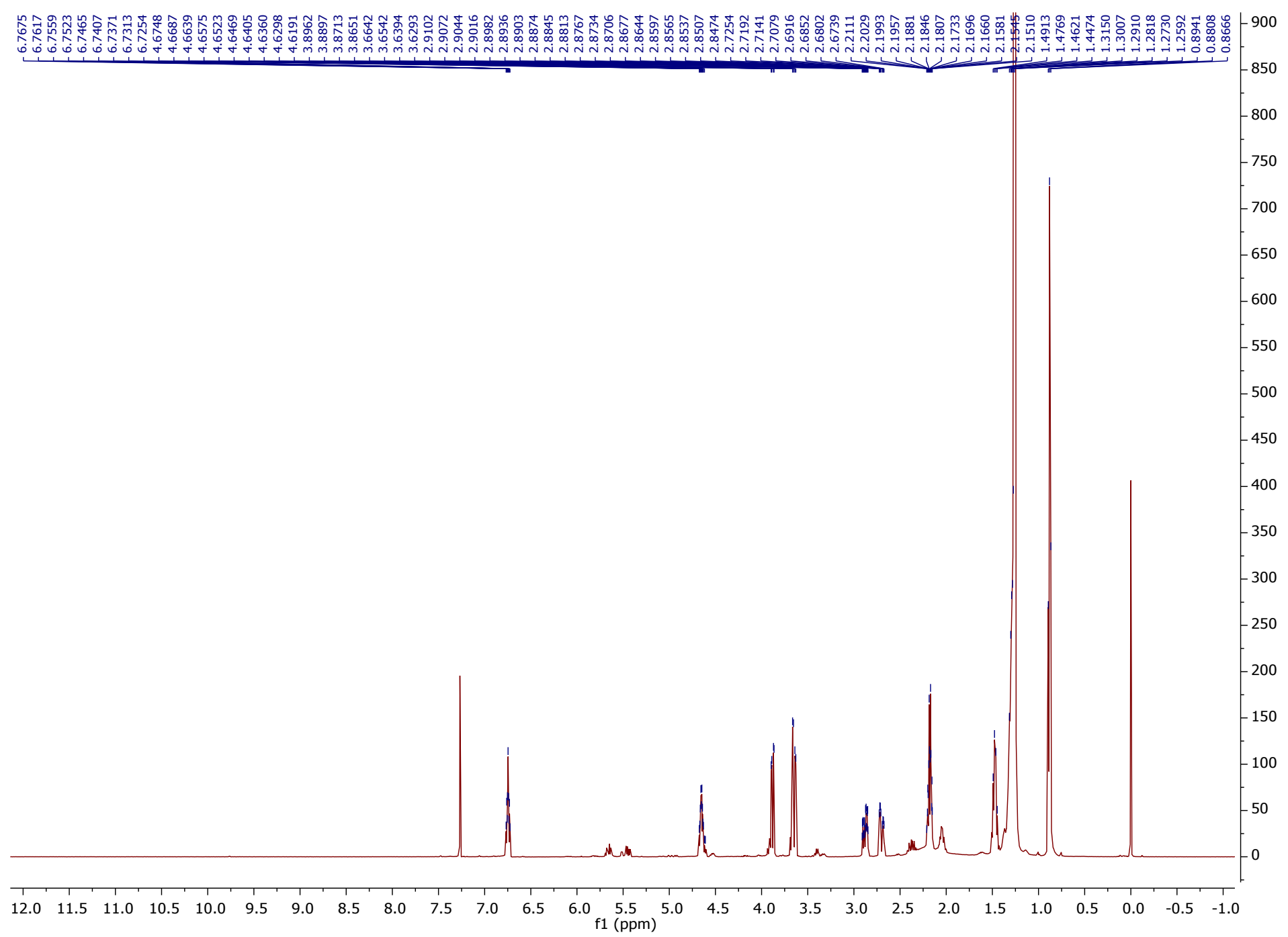

Figure S18. ${ }^{1} \mathrm{H}$ NMR spectrum $\left(500 \mathrm{MHz}, \mathrm{CDCl}_{3}\right)$ of compound 3 . 


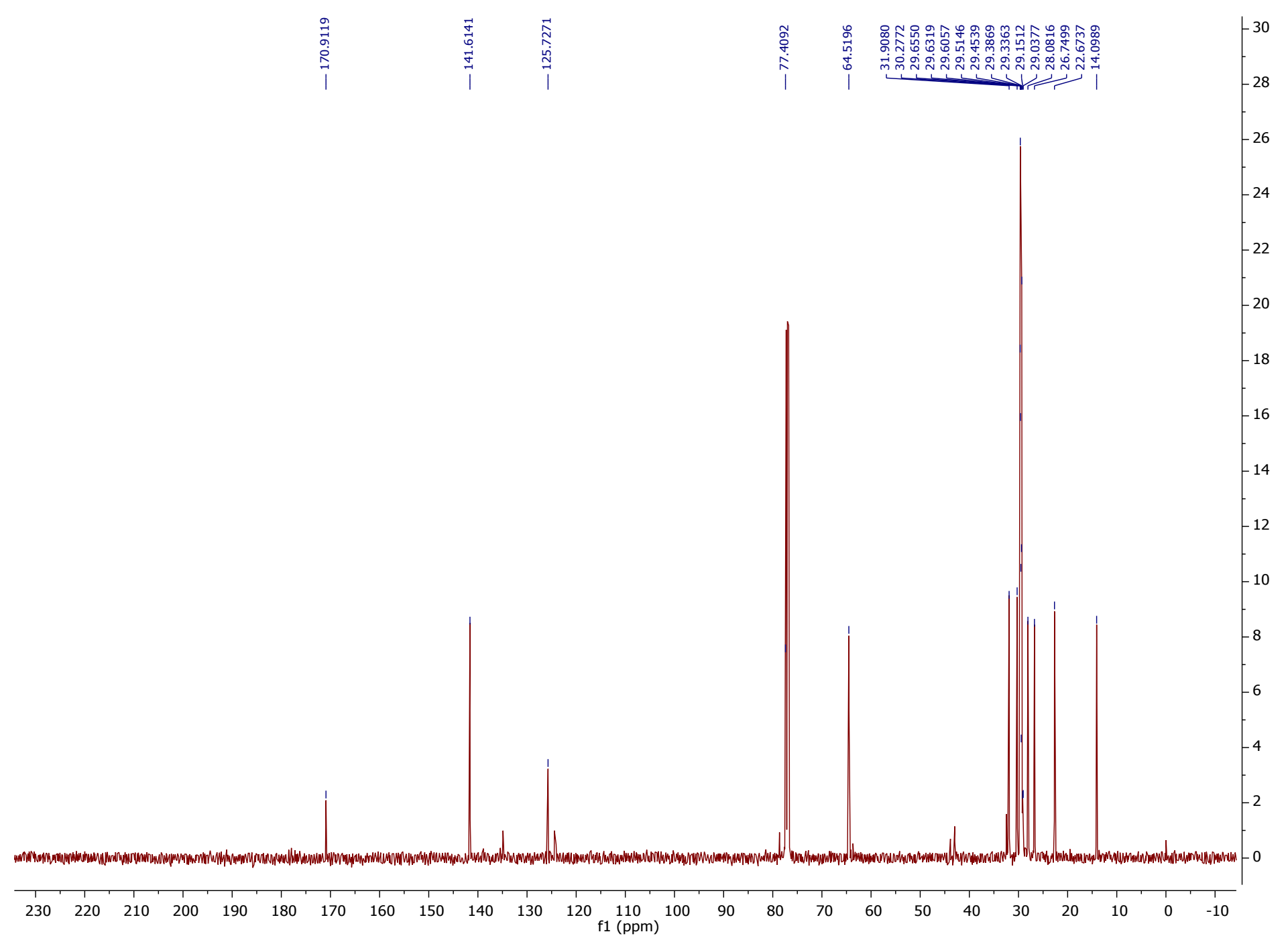

Figure S19. ${ }^{13} \mathrm{C}$ NMR spectrum $\left(125 \mathrm{MHz}, \mathrm{CDCl}_{3}\right)$ of compound 3. 


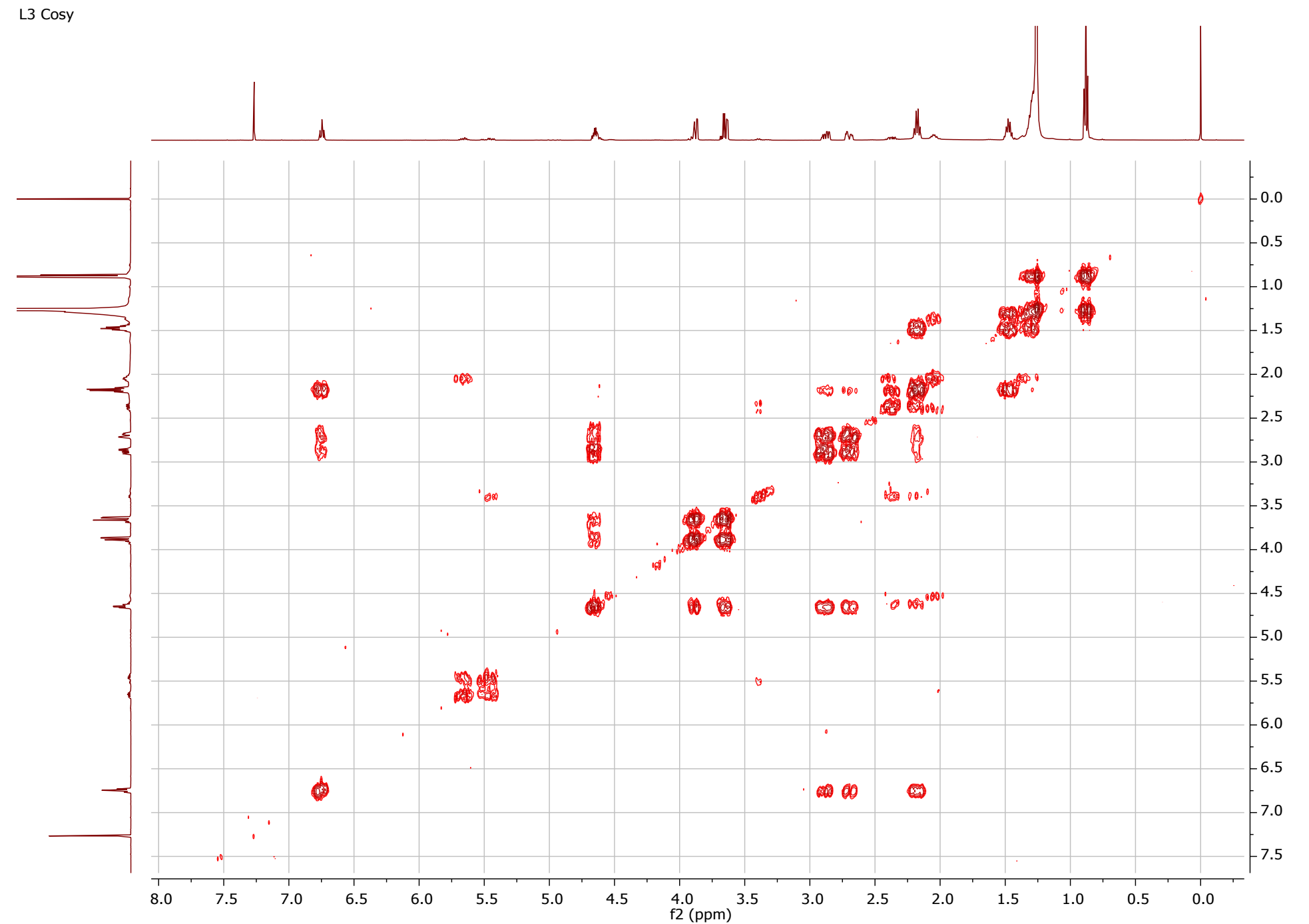
$\underset{\substack{\underline{0}\\}}{\bar{c}}$

Figure S20. COSY spectrum $\left(500 \mathrm{MHz}, \mathrm{CDCl}_{3}\right)$ of compound 3. 


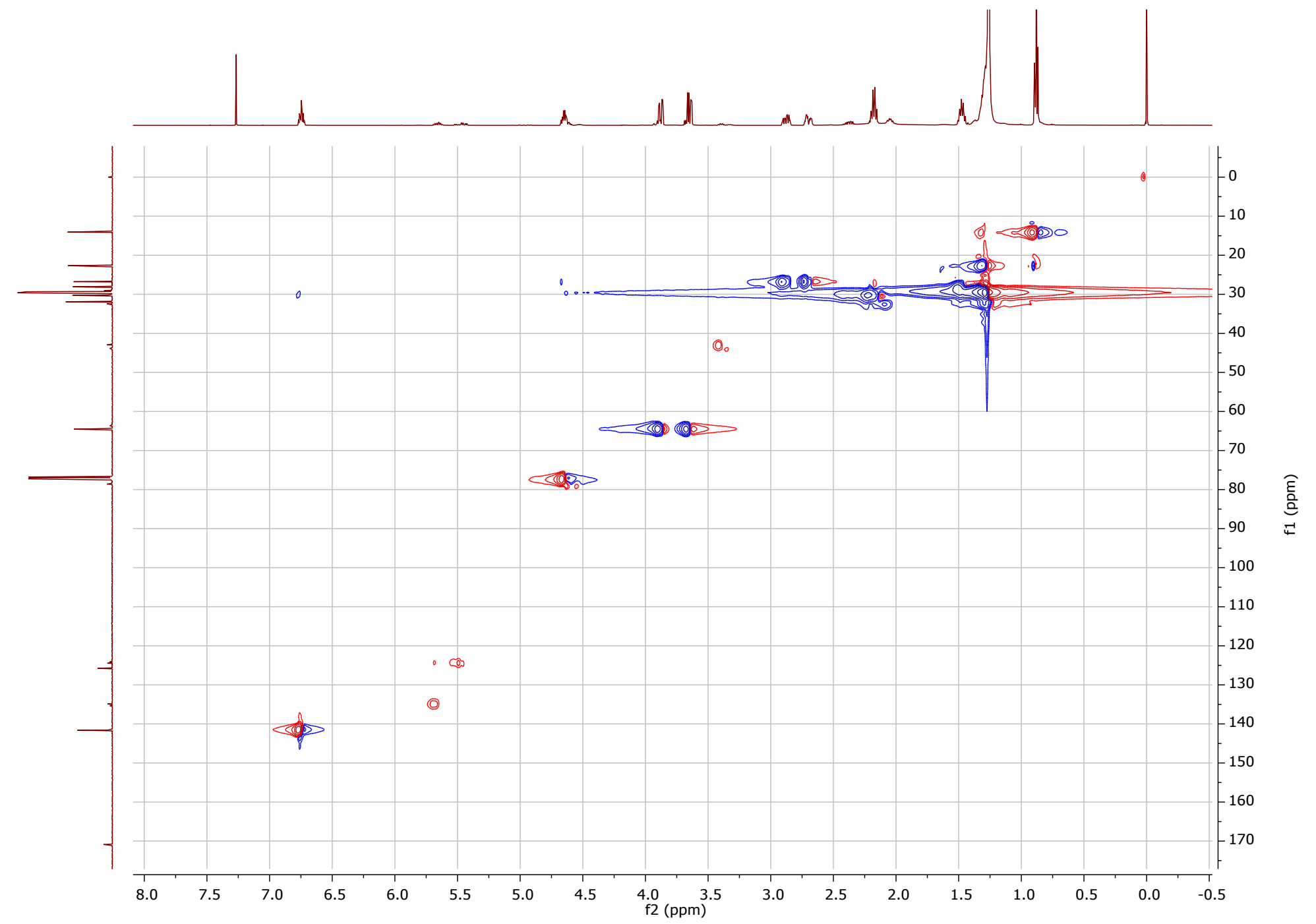

Figure S21. HSQC spectrum (500 and $125 \mathrm{MHz}, \mathrm{CDCl}_{3}$ ) of compound 3. 


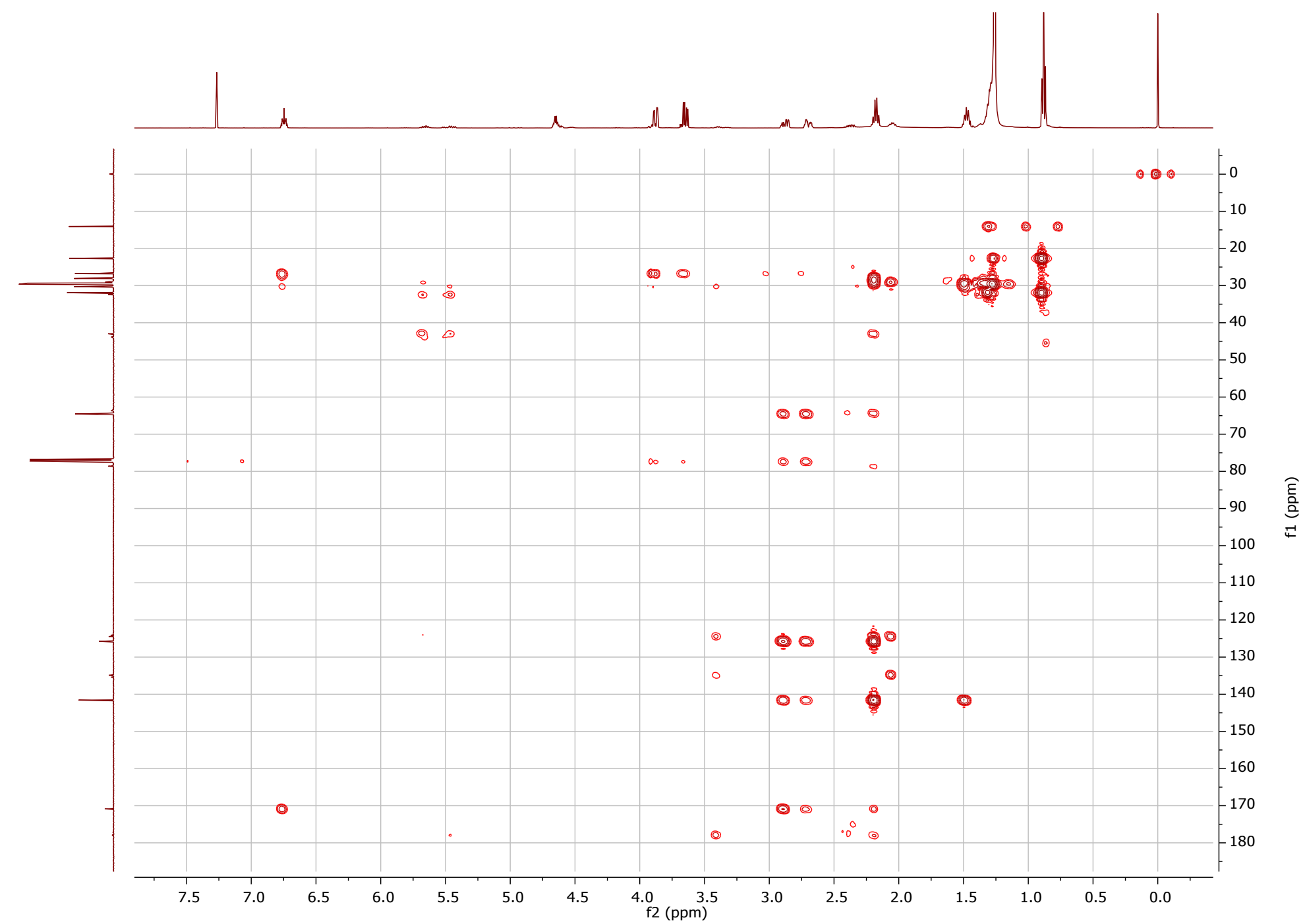

Figure S22. $\mathrm{HMBC}$ spectrum (500 and $125 \mathrm{MHz}, \mathrm{CDCl}_{3}$ ) of compound 3. 


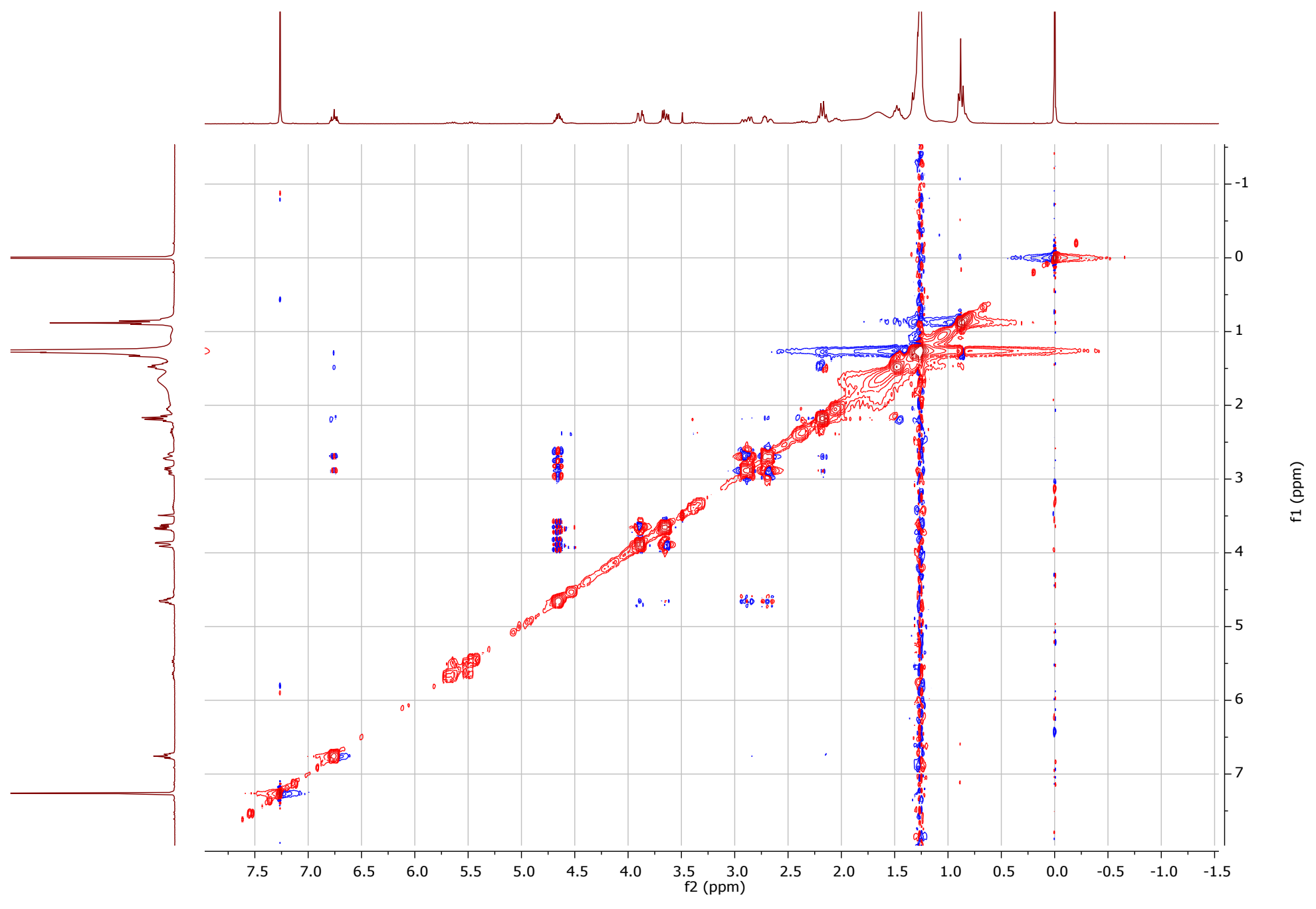

Figure S23. NOESY spectrum (500 MHz, $\left.\mathrm{CDCl}_{3}\right)$ of compound 3. 


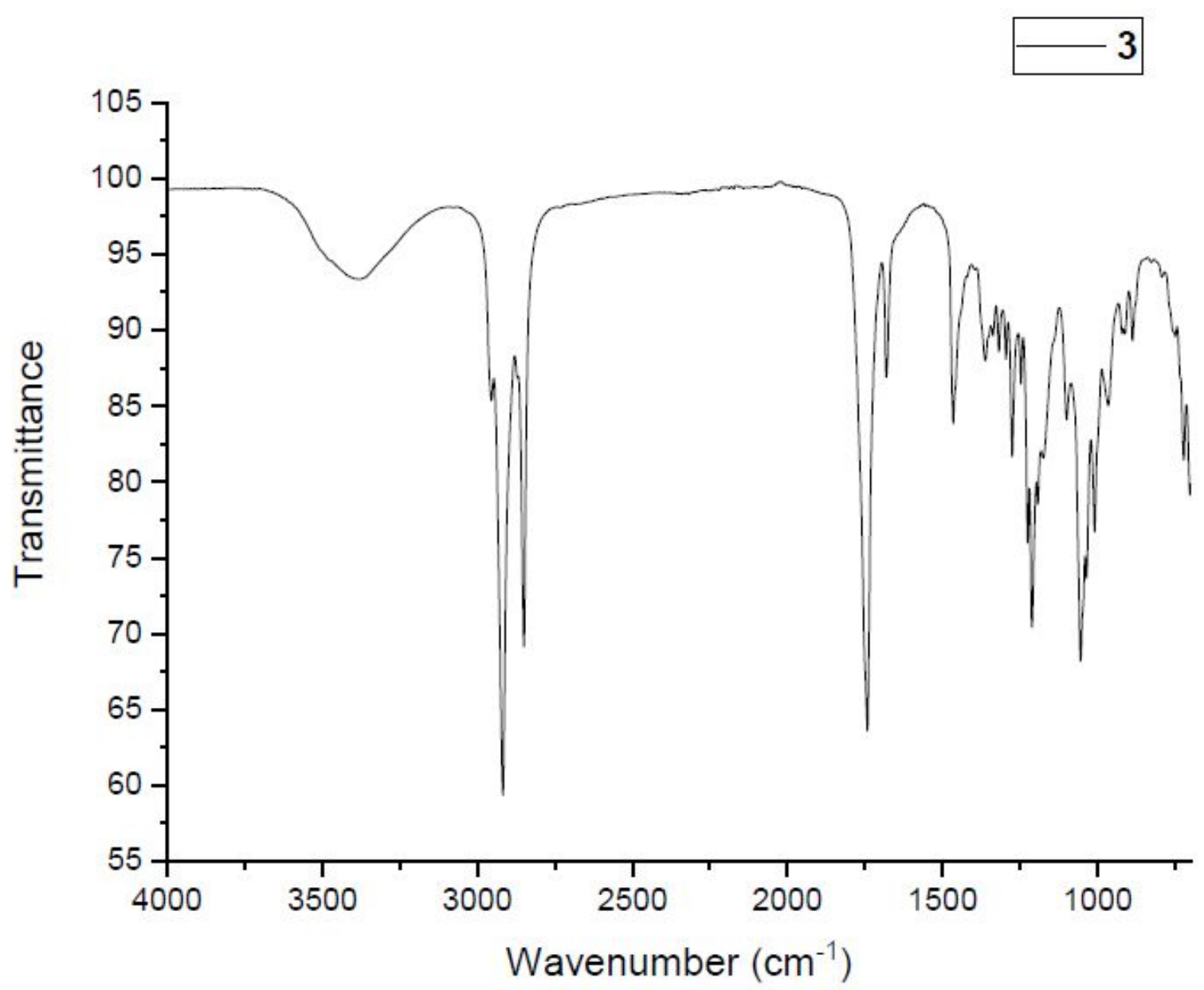

Figure S24. Infrared (IR) spectrum of compound 3. 


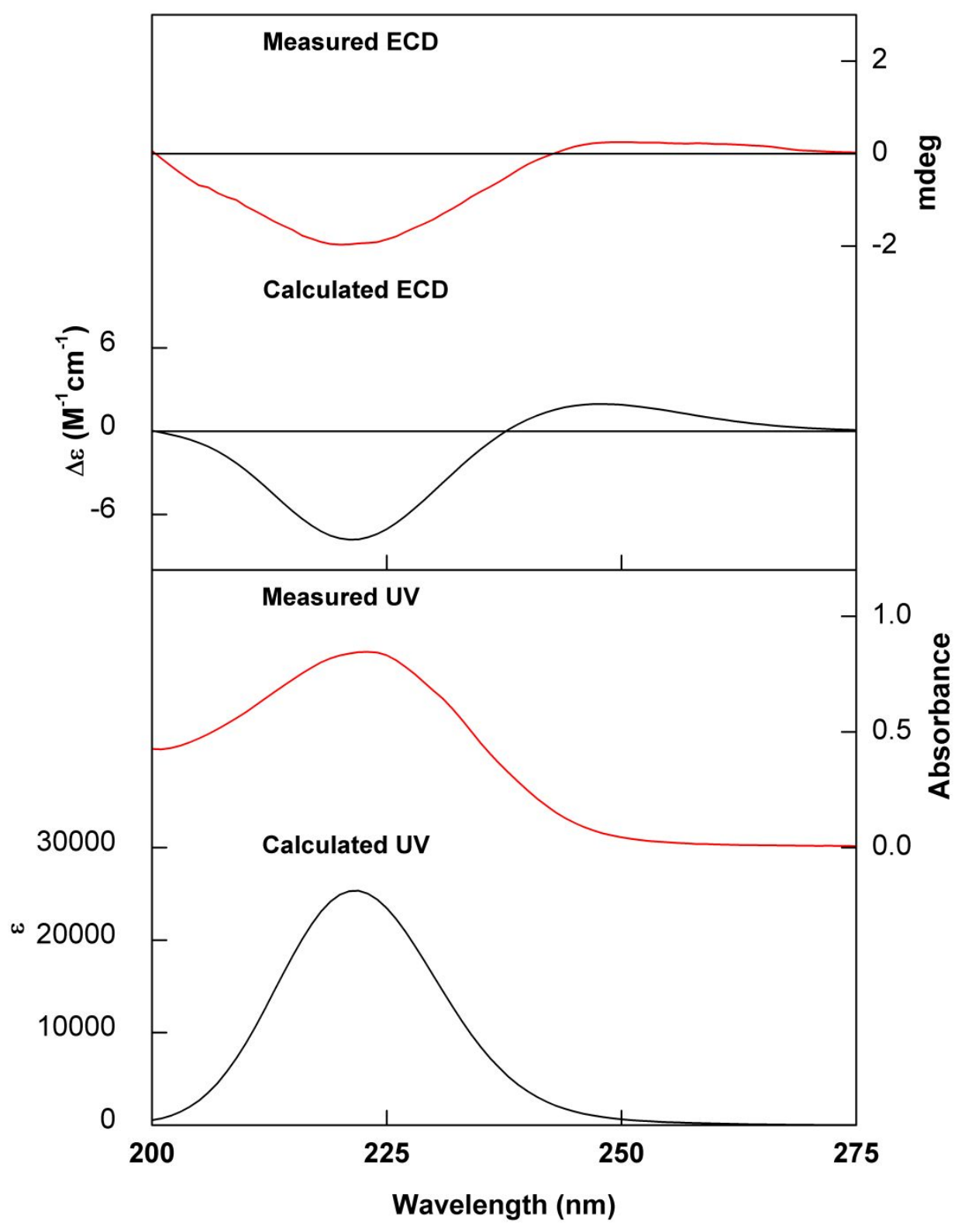

Figure S25. Comparison of the observed UV and ECD spectra of (-)-2 (red trace), recorded in $\mathrm{MeOH}$, with the calculated [CAMB3LYP/PCM(MeOH)/TZVP//B3PW91/PCM(MeOH)/6-311G(d,p)] UV and ECD spectra (black trace) of the simple average of the lowest-energy conformers identified for the $(R)-\mathbf{2}$. 


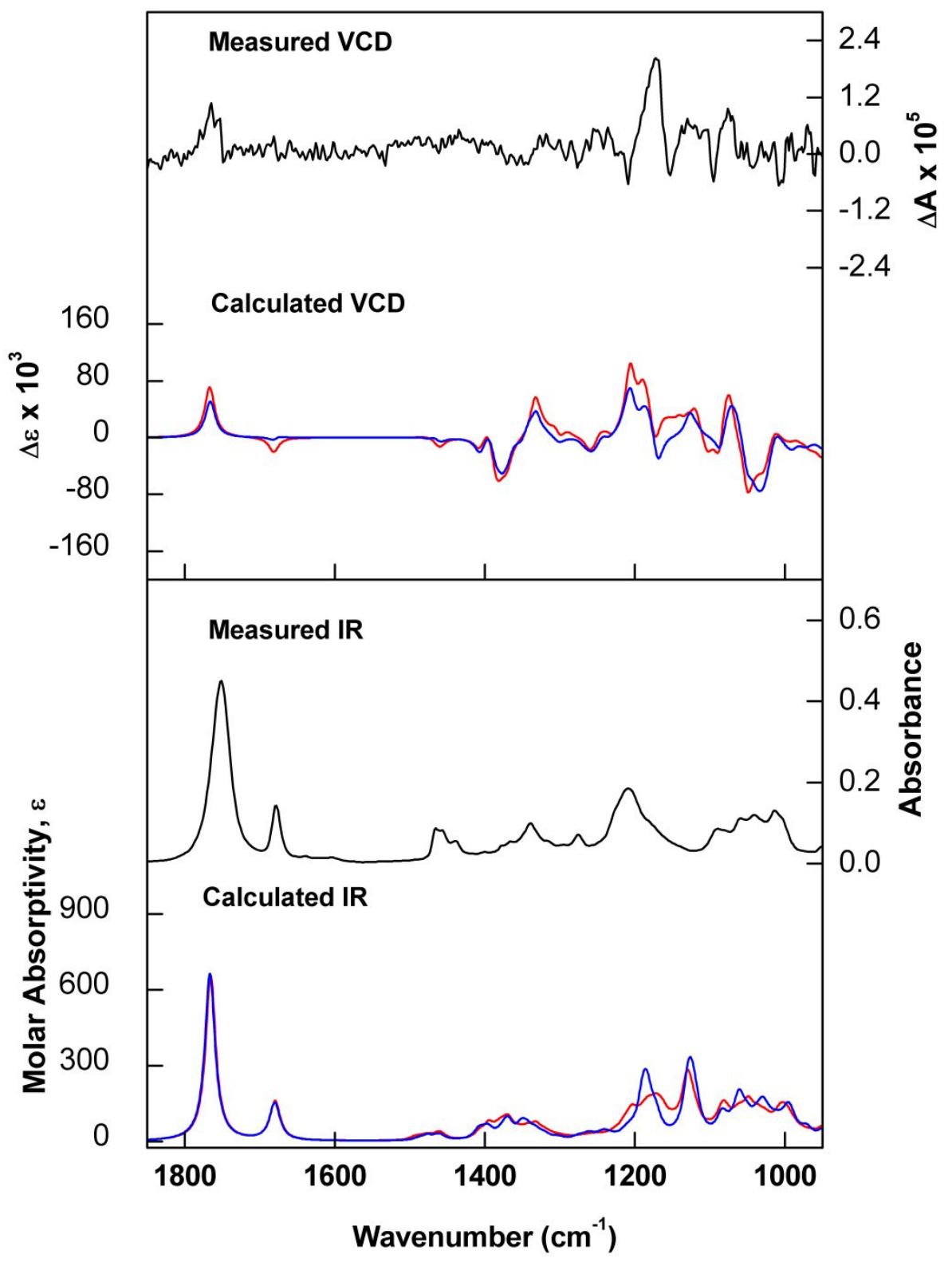

Figure S26. Comparison of the observed IR and VCD spectra of (-)-2 (black trace), recorded in $\mathrm{CDCl}_{3}$, with the calculated [B3LYP/PCM $\left.\left(\mathrm{CHCl}_{3}\right) / 6-31 \mathrm{G}(\mathrm{d})\right]$ IR and VCD spectra of the simple average of the lowest-energy conformers identified for the $(R)$-truncated model (blue trace) and $(R)-2$ (red trace). 


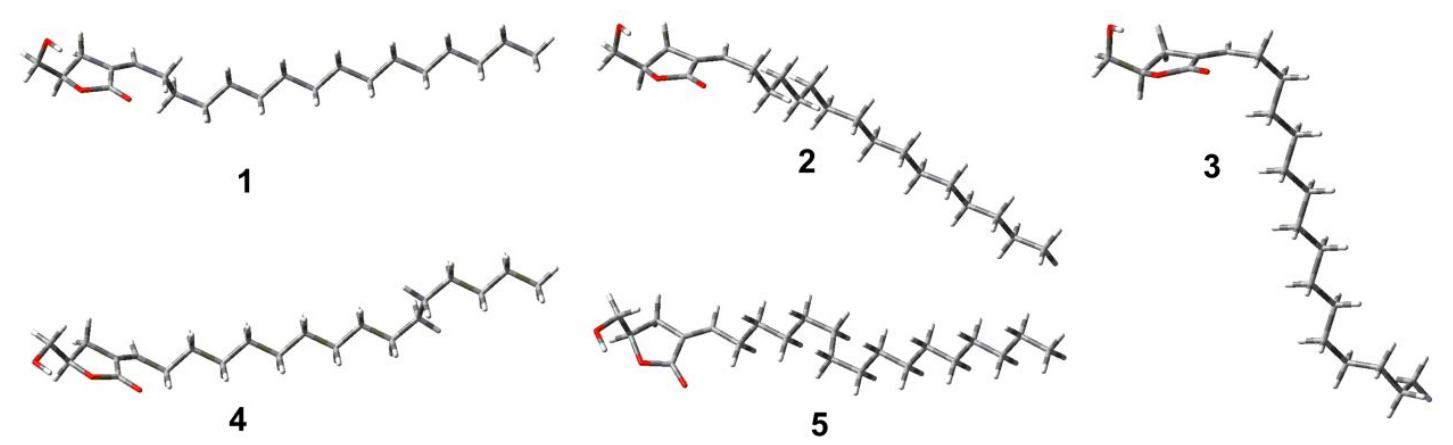

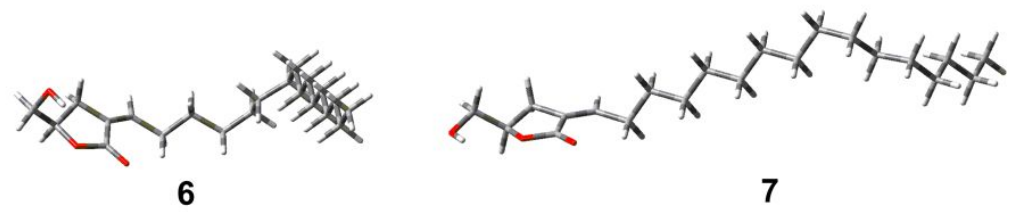<smiles>CC(=CC(C)C(C)C(C)C(C)C(C)C(C)C(C)C(C)C(C)C(C)C(C)C(C)C(C)(C)C(C)(C)C(C)C)C(C)C</smiles>

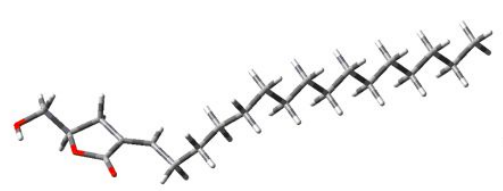

10

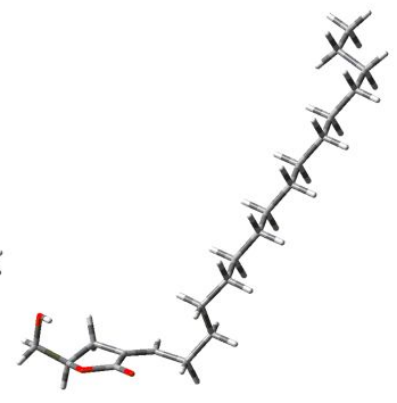

8

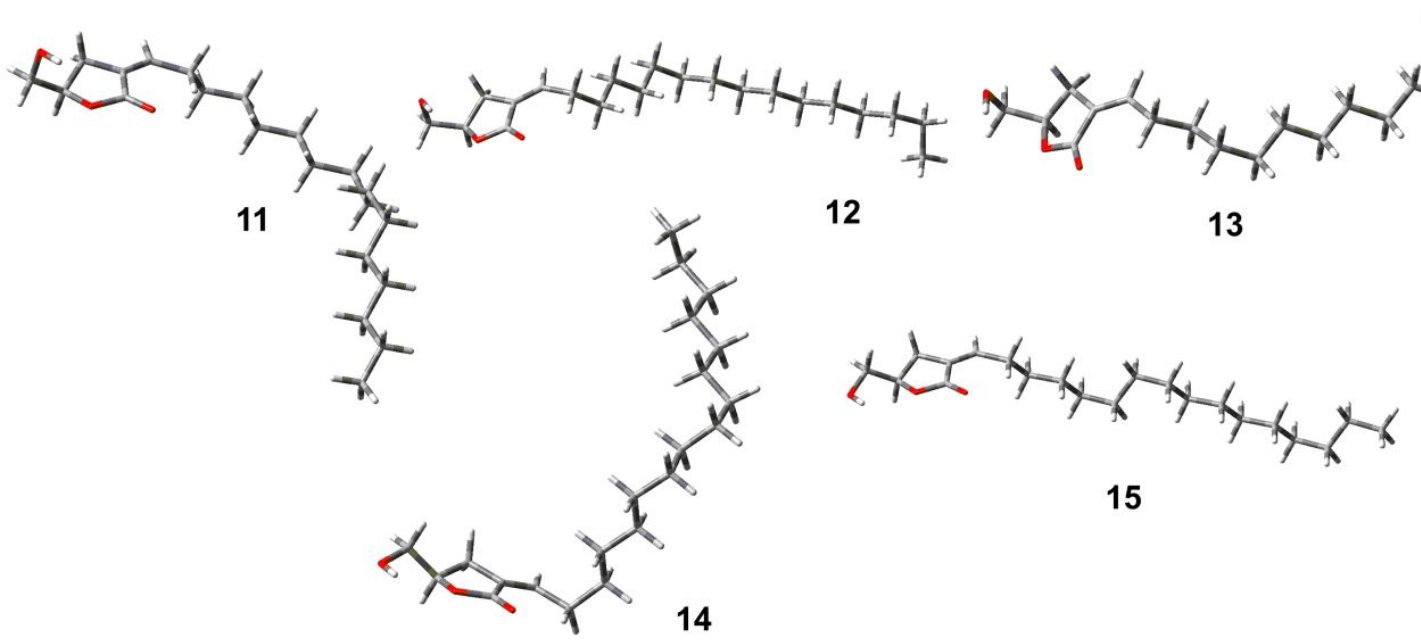

Figure S27. Optimized structures of the lowest-energy conformers identified for $(R)-2$ at the B3LYP/PCM $\left(\mathrm{CHCl}_{3}\right) / 6-31 \mathrm{G}(\mathrm{d})$ level and used as a simple average. 


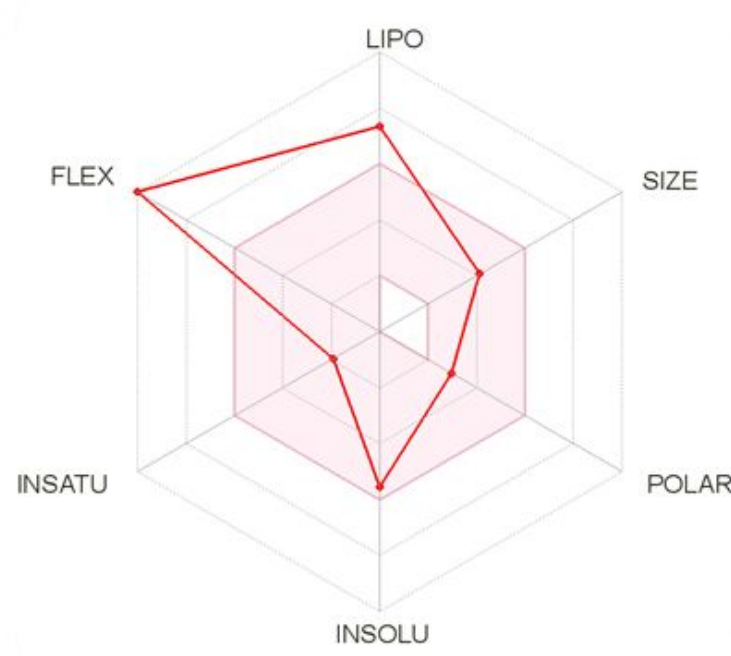

1

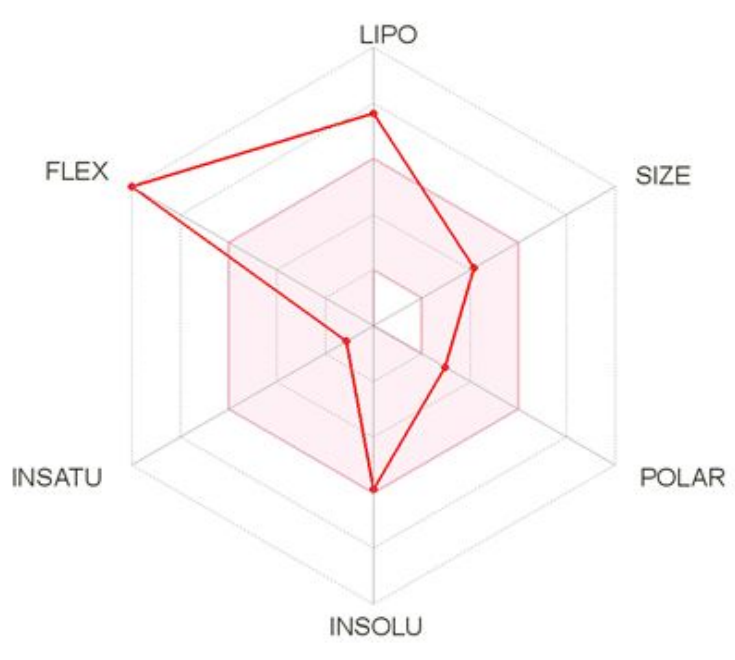

2

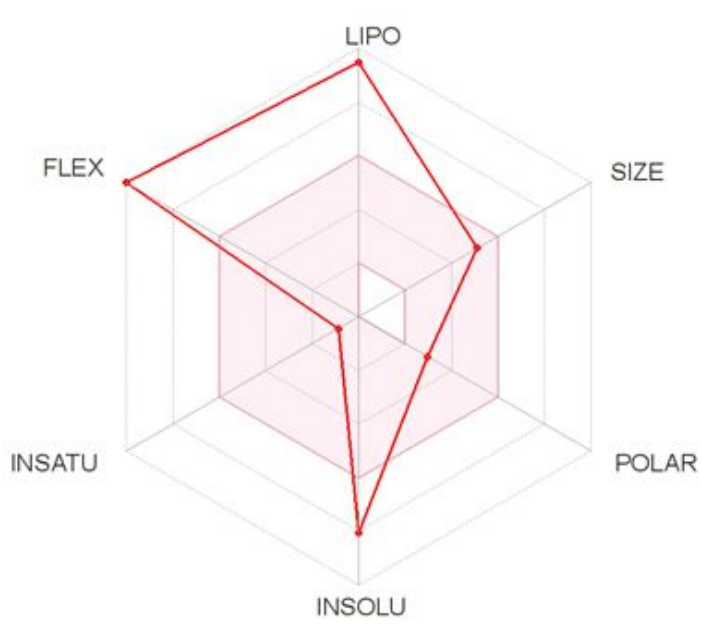

3

Figure S28. Drug-likeness of compounds $1-\mathbf{3}$ using the Bioavailability Radar (SwissADME). The pink area represents the optimal range for each properties. 
Table S1. Thermochemical analysis of the $(R)$-truncated model.

\begin{tabular}{|c|c|c|c|c|}
\hline \multirow[t]{2}{*}{ Conformer } & \multicolumn{2}{|c|}{ B3PW91/PCM(MeOH)/6-311G(d,p) } & \multicolumn{2}{|c|}{ B3LYP/PCM(CHCl 3 )/6-31G(d) } \\
\hline & $\Delta \mathbf{G}(\mathrm{kcal} / \mathrm{mol})$ & $\begin{array}{c}\text { Boltzmann } \\
\text { population \% }\end{array}$ & $\Delta \mathbf{G}(\mathrm{kcal} / \mathrm{mol})$ & $\begin{array}{c}\text { Boltzmann } \\
\text { population } \%\end{array}$ \\
\hline 1 & 0 & 15 & 0 & 15 \\
\hline 2 & 0.09 & 13 & 0.09 & 13 \\
\hline 3 & 0.22 & 10 & 0.18 & 11 \\
\hline 4 & 0.26 & 10 & 0.17 & 12 \\
\hline 5 & 0.43 & 7 & 0.42 & 8 \\
\hline 6 & 0.44 & 7 & 0.62 & 5 \\
\hline 7 & 0.51 & 6 & 0.53 & 6 \\
\hline 8 & 0.58 & 5 & 0.41 & 8 \\
\hline 9 & 0.61 & 5 & 0.51 & 6 \\
\hline 10 & 0.73 & 4 & 1.05 & 3 \\
\hline 11 & 0.85 & 4 & 0.99 & 3 \\
\hline 12 & 0.90 & 3 & 1.05 & 3 \\
\hline 13 & 0.97 & 3 & - & - \\
\hline 14 & 1.12 & 2 & 0.87 & 4 \\
\hline 15 & 1.14 & 2 & - & - \\
\hline 16 & 1.15 & 2 & 0.94 & 3 \\
\hline 17 & 1.17 & 2 & - & - \\
\hline
\end{tabular}


Table S2. Thermochemical analysis of (R)-2.

\begin{tabular}{|c|c|c|c|c|}
\hline \multirow[t]{2}{*}{ Conformer } & \multicolumn{2}{|c|}{ B3PW91/PCM(MeOH)/6-311G(d,p) } & \multicolumn{2}{|c|}{ B3LYP/PCM $\left(\mathrm{CHCl}_{3}\right) / 6-31 \mathrm{G}(\mathrm{d})$} \\
\hline & $\Delta \mathbf{G}(\mathrm{kcal} / \mathrm{mol})$ & $\begin{array}{c}\text { Boltzmann } \\
\text { population \% }\end{array}$ & $\Delta \mathbf{G}(\mathrm{kcal} / \mathrm{mol})$ & $\begin{array}{c}\text { Boltzmann } \\
\text { population } \%\end{array}$ \\
\hline 1 & 0 & 60 & 0 & 17 \\
\hline 2 & 1.01 & 11 & 0.61 & 6 \\
\hline 3 & 1.41 & 6 & 0.79 & 4 \\
\hline 4 & 1.49 & 5 & 0.41 & 8 \\
\hline 5 & 1.53 & 5 & 0.09 & 14 \\
\hline 6 & 1.6 & 4 & 0.71 & 5 \\
\hline 7 & 1.7 & 3 & 0.39 & 9 \\
\hline 8 & 1.91 & 2 & 0.92 & 3 \\
\hline 9 & 1.95 & 2 & 0.32 & 10 \\
\hline 10 & 1.98 & 2 & 0.53 & 7 \\
\hline 11 & - & - & 0.58 & 6 \\
\hline 12 & - & - & 0.97 & 3 \\
\hline 13 & - & - & 1.00 & 3 \\
\hline 14 & - & - & 1.05 & 3 \\
\hline 15 & - & - & 1.27 & 2 \\
\hline
\end{tabular}


Table S3. In silico predictions of ADME and drug-likeness of compounds 1 3 using SwissADME platform.

\begin{tabular}{lll}
\hline 1 & 2 & 3
\end{tabular}

Physicochemical proprieties

\begin{tabular}{cccc}
\hline MW & $336.51 \mathrm{Da}$ & $338.52 \mathrm{Da}$ & $422.68 \mathrm{Da}$ \\
Number of heavy atoms & 24 & 24 & 30 \\
Num. aromomatic heavy atoms & 0 & 0 & 0 \\
Fraction Csp & & 0.86 & 0.89 \\
Number of rotatable bounds & 15 & 15 & 21 \\
Number of H-bond acceptors & 3 & 3 & 3 \\
Number of H-bound donnors & 1 & 1 & 1 \\
Log Po/w & 5.54 & 5.78 & 7.88 \\
Water solubility & moderate & poor & Poor
\end{tabular}

Pharmacokinetics

\begin{tabular}{cccc}
\hline $\begin{array}{c}\text { Gl absorption } \\
\text { BBB }\end{array}$ & High & High & Low \\
permeability & Yes & Yes & No \\
P-gp & No & No & Yes \\
substrate & & & \\
CYP 1A2 & Yes & Yes & Yes \\
inhibitor & & & \\
CYP2C19 & Yes & Yes & No \\
inhibitor & & &
\end{tabular}




\begin{tabular}{llcc}
\hline CYP2C9 & Yes & Yes & No \\
inhibitor & & No & No \\
CYP2D6 & No & & \\
inhibitor & & No & No \\
CYP3A4 & No & & \\
inhibitor & & & \\
\hline
\end{tabular}

\section{Druglikeness}

Lipinski yes; 0 violation yes; 0 violation $\quad$ yes; 1 violation: mlogP

$$
>4.15
$$

Ghose $\quad$ Yes no; 1 violation: no; 3 violations: wlogP

$$
\begin{aligned}
\text { wlog } P>5.6 & >5.6, \mathrm{mr}>130, \\
& \text { \#atoms }>70
\end{aligned}
$$

Veber no; 1 violation: rotors no; 1 violation: no; 1 violation: rotors

$\begin{array}{cccc} & >10 & \text { rotors }>10 & >10 \\ \text { Egan } & \text { Yes } & \text { Yes } & \text { no; } 1 \text { violation: wlog } P \\ & & >5.88\end{array}$

Muegge no; 1 violation: no; 1 violation: no; 2 violations: $x \log P 3$

$$
\mathrm{x} \log \mathrm{P} 3>5 \quad \mathrm{x} \log \mathrm{P} 3>5 \quad>5, \text { rotors }>15
$$

Medicinal Chemistry

\begin{tabular}{cccc}
\hline PAINS & no alert & no alert & no alert \\
Brenk & 2 alerts: isolated & 1 alert: Michael & 1 alert: Michael \\
& alkene, Michael & acceptor & acceptor \\
& acceptor & & \\
Leadlikeness & No & No & No \\
\hline
\end{tabular}

